\title{
InVESTIGATION OF AGENT-BASEd MODELLING FOR INPATIENT HEALTHCARE SYSTEMS
}

\author{
by \\ Amirkiarash KIANI, B.Sc. \\ University of Borås, Borås, Sweden, 2012
}

\begin{abstract}
A thesis presented to Ryerson University
in partial fulfilment of the requirement for the degree of

Master of Applied Science in the Program of

Mechanical and Industrial Engineering
\end{abstract}

Toronto, Ontario, Canada, 2016

(c) 2016 Amirkiarash Kiani 


\section{Author's Declaration}

I hereby declare that I am the sole author of this thesis. This is a true copy of the thesis, including any required final revisions, as accepted by my examiners.

I authorize Ryerson University to lend this thesis to other institutions or individuals for the purpose of scholarly research.

I further authorize Ryerson University to reproduce this thesis by photocopying or by other means, in total or in part, at the request of other institutions or individuals for the purpose of scholarly research.

I understand that my thesis may be made electronically available to the public. 


\title{
INVESTIGATION OF AGENT-BASED MODELLING FOR INPATIENT HEALTHCARE SYSTEMS
}

\author{
Amirkiarash Kiani \\ Master of Applied Science - 2016 \\ Industrial and Mechanical Engineering \\ Ryerson University
}

\begin{abstract}
The goal of this research was to investigate the possibility of using Agent-based Modelling, a novel approach in computerized simulation, to assess the effects of staff ratio on recovery time and to develop an empirical research plan based on an inpatient unit. By creating a virtual unit, the researcher was able to develop an adjustable model to test several scenarios based on empirical evidence; to comprehend the impact of changes to staff ratio and patient acuity on nurses' workload and quality of care to patients. This investigation found that acuity indices of patients have no significant effect on available recovery time or the number of unperformed activities. On the contrary, nurse/patient ratio has substantial effects on both available recovery time and the number of unperformed activities; which asserts the significant effect of insufficient nurse staffing on the well-being of nurses as well as quality of care to patients.
\end{abstract}




\section{Acknowledgements}

I would like to express my sincere gratitude to my supervisors Drs Patrick Neumann and Nancy Purdy who have been a strong source of inspiration throughout my project work. Besides my supervisors, I would like to thank the rest of my thesis committee: Dr Mohamed Wahab Mohamed Ismail and Dr Cory Searcy, for their insightful comments and feedback. I would also like to place on record my gratitude to the Graduate Program Director of Mechanical and Industrial Engineering Department: Dr Ahmad Ghasempoor for his support. I would like to thank my family: Rezvan, Bakhtiar, Anahita, Kianoosh, Mehrnaz, Adrian, and Elliot. Thanks for your continual support and listening, which has given me the ability to pursue my goals with confidence and full determination.

I would like to acknowledge Drs Sharareh Taghipour, Vinh Quan, and Vincent Chan who provided me with an enhanced educational experience which I will never forget. I thank Dr Ozgur Turetken for all the encouragements, understanding, and the occasional laughs. Last but not least, I'd like to mention Dr Jan H van Vuuren who has had the most influence on my academic journey and pursuing graduate studies. Thanks for a wide range of conversations, from P. Blackett to M. Bulgakov, and hope to watch Puccini's Tosca together again soon. 


\section{Dedication}

To my best friend, idol, and only brother: Kianoosh. 


\section{Table of Contents}

Declaration $\quad$ ii

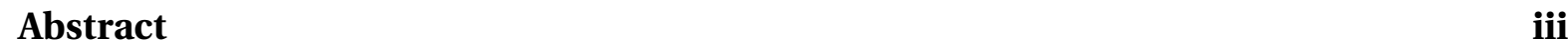

Acknowledgements $\quad$ iv

Dedication $\quad$ v

$\begin{array}{ll}\text { List of Tables } & \mathbf{x}\end{array}$

List of Figures $\quad$ xii

List of Algorithms $\quad$ xiii

List of Abbreviations $\quad$ xiv

1 Introduction $\quad 1$

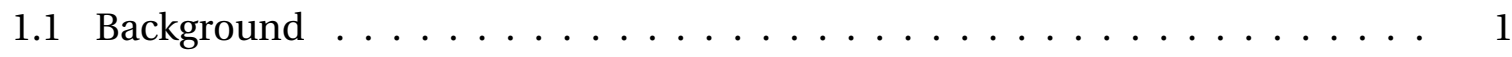

1.1.1 Emphasis on Emergen'cy Departments . . . . . . . . . . . 7

1.1.2 Ignoring Nurse/Patient Ratios $\ldots \ldots \ldots \ldots$

1.1.3 Ignoring Patient Acuity Index $\ldots \ldots \ldots \ldots$

1.2 Research Questions $\ldots \ldots \ldots \ldots \ldots$ 
2 Method 10

2.1 Study Design and Data Collection $\ldots \ldots \ldots \ldots \ldots$

2.1 .1 Sample and Setting . . . . . . . . . . . . . . . . . 10

2.1.2 Gaining Access to Participants and Quantitative Data . . . . . . . . 11

2.1.3 Interviews with the GRASP Manager . . . . . . . . . . . . . . . . . . 12

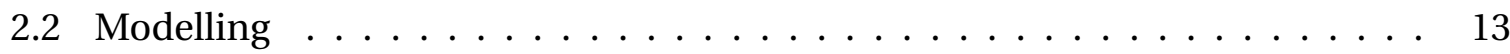

2.2 .1 ABM Software Selection . . . . . . . . . . . . . . . 13

2.2.2 Model Conceptualization . . . . . . . . . . . . . . . 13

2.3 Data Analysis Plan . . . . . . . . . . . . . . . . . . . . 19

2.4 Verification and Validation $\ldots \ldots \ldots \ldots \ldots \ldots$

2.5 Data Mining of GRASP Data . . . . . . . . . . . . . . . . . 22

2.5.1 Experimental Design $\ldots \ldots \ldots \ldots \ldots \ldots \ldots$

3 Results $\quad 25$

3.1 RQ1: ABM and the Virtual Unit . . . . . . . . . . . . . . . . . . 25

3.2 Experimental Design Results $\ldots \ldots \ldots \ldots \ldots \ldots$

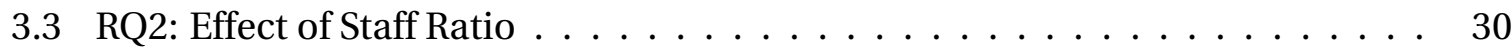

3.3.1 Effects on Available Recovery Time $(\%) \ldots \ldots \ldots$

3.3.2 Effects on Number of Unperformed Activities _ . . . . . . . . . . 31

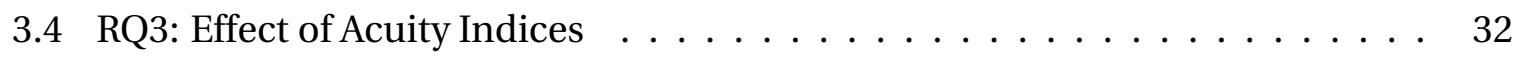

3.4.1 Effects on Available Recovery Time $(\%) \ldots \ldots \ldots$. . . . . . . . . 32

3.4.2 Effects on Number of Unperformed Activities . . . . . . . . . . . . . 33

4 Discussion $\quad 34$

4.1 RQ1: ABM and the Virtual Unit . . . . . . . . . . . . . . . . . . . 34

4.2 RQ2: Effects of Staff Ratio $\ldots \ldots \ldots \ldots \ldots \ldots \ldots \ldots$

4.3 RQ3: Effects of Acuity Indices . . . . . . . . . . . . . . . . . . . . 37 
4.4 Methodological Issues and Limitations $\ldots \ldots \ldots \ldots$

5 Conclusion $\quad 39$

5.1 Contributions ........................ 40

5.2 Future Studies . . . . . . . . . . . . . . . . . . 40

\section{Appendices}

$\begin{array}{ll}\text { A Pseudocodes } & 43\end{array}$

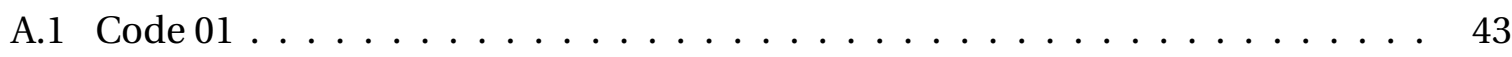

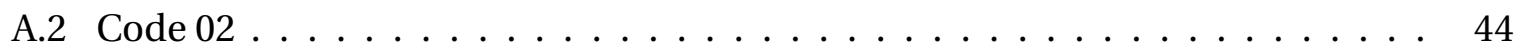

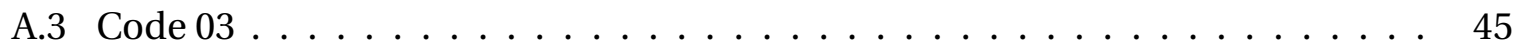

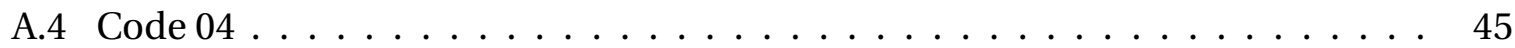

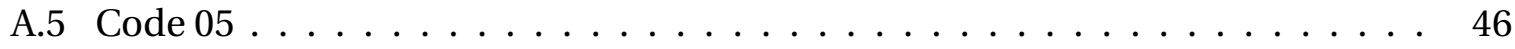

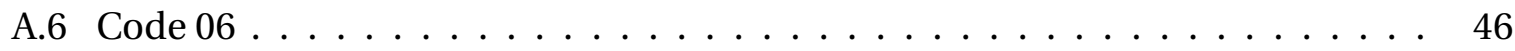

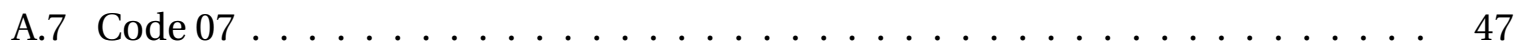

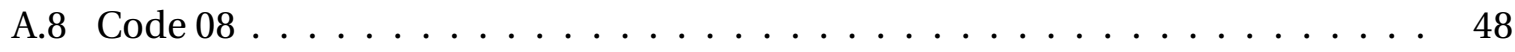

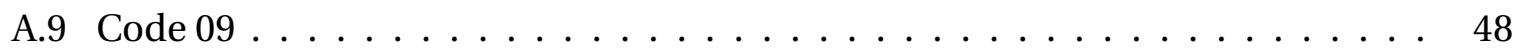

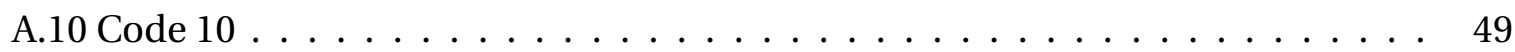

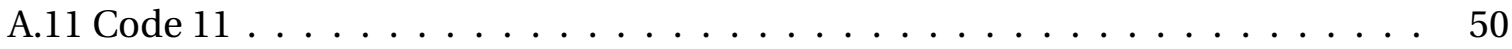

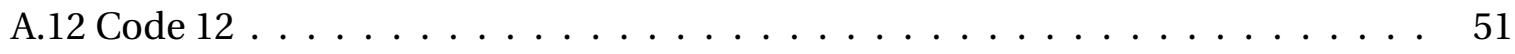

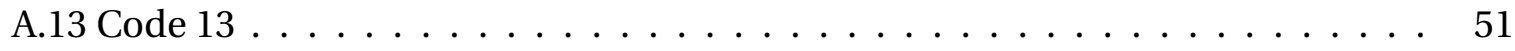

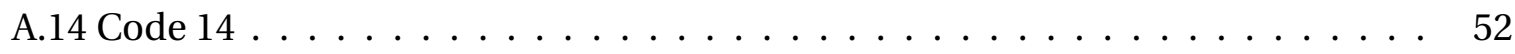

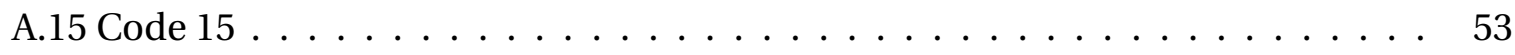

A.16 Code $16 \ldots \ldots \ldots \ldots \ldots \ldots \ldots \ldots \ldots \ldots$

$\begin{array}{ll}\text { B Output Analysis } & 55\end{array}$

$\begin{array}{ll}\text { References } & 66\end{array}$ 


\section{List of Tables}

1.1 Prominent characteristics of ABM in comparison to DES . . . . . . . . . 5

1.2 Current trends and found gaps in ABM and Healthcare studies $\ldots \ldots \ldots$

2.1 List of activities and their descriptions in the selected unit $\ldots \ldots$. . . . . 18

2.2 Example of GRASP data received from the selected unit . . . . . . . . . . 22

2.3 Calculated durations (weighted arithmetic means) of considered activities . 23

2.4 Matrix of nine defined tests . . . . . . . . . . . . . . . . . . . . 24

3.1 Results of available recovery time (\%) and average number of unperformed activities for the ten replications of each condition $\ldots \ldots \ldots$

B.1 The minimum number of replications $\ldots \ldots \ldots \ldots \ldots \ldots$

B.2 High \& High - Available Recovery Time $(\%) \ldots \ldots \ldots$

B.3 High \& Medium - Available Recovery Time $(\%) \ldots \ldots \ldots$

B.4 High \& Low - Available Recovery Time (\%) . . . . . . . . . . . . . . . . 56

B.5 Medium \& High - Available Recovery Time $(\%) \ldots \ldots$. . . . . . . . . . . 56

B.6 Medium \& Medium - Available Recovery Time (\%) f . . . . . . . . . . . 56

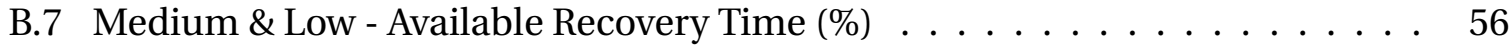

B.8 Low \& High - Available Recovery Time $(\%) \ldots \ldots \ldots \ldots$

B.9 Low \& Medium - Available Recovery Time (\%) . . . . . . . . . . . . . . 57 
B.10 Low \& Low - Available Recovery Time $(\%) \ldots \ldots \ldots 7$ 


\section{List of Figures}

1.1 Aims in alteration of healthcare units and organizations . . . . . . . . . 3

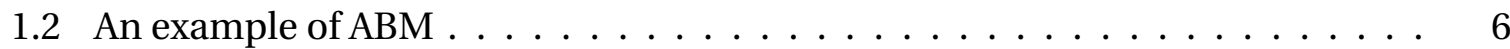

2.1 Flowchart of nurses in the virtual unit . . . . . . . . . . . . . . . 15

2.2 Flowchart of patients in the virtual unit . . . . . . . . . . . . . . 15

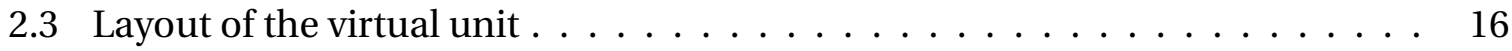

2.4 Details of rooms and nurse station $\ldots \ldots \ldots \ldots \ldots \ldots$

2.5 Considered activities for nurses $\ldots \ldots \ldots \ldots \ldots \ldots \ldots$

3.1 Example of movements of agents in the virtual unit: three-dimensional and spaghetti plots respectively $\ldots \ldots \ldots \ldots \ldots$

3.2 Tools developed for altering independent variables during computerized simu-

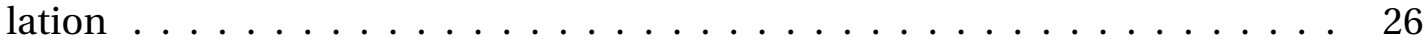

3.3 Time-stack and time plot charts for ratios of one nurse to nine patients (top) and one nurse to three patients (bottom) $\ldots \ldots \ldots \ldots \ldots$

3.4 Three-dimensional plot of results of two responses: available recovery time and number of unperformed activities $\ldots \ldots \ldots \ldots$

3.5 Box plots of results of two responses: available recovery time and number of unperformed activities respectively $\ldots \ldots \ldots$. . . . . . . . . . 29 
3.6 Bar charts with error bars $(\alpha=5 \%)$ : available recovery time, and number of unperformed activities $\ldots \ldots \ldots \ldots \ldots \ldots$

3.7 Plot of one-way ANOVA ( $\alpha=5 \%$ ): available recovery (response), and staff ratio 30

3.8 Plot of post-hoc analysis: effects of staff ratio on available recovery time $\ldots 30$

3.9 Plot of one-way ANOVA: number of unperformed activities, and staff ratio . . 31

3.10 Plot of post-hoc analysis: effects of staff ratio on number of unperformed

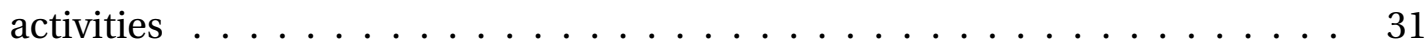

3.11 Plot of one-way ANOVA: available recovery time, and acuity $\ldots \ldots$. . . . . 32

3.12 Plot of post-hoc analysis: effects of acuity indices on available recovery time . 32

3.13 Plot of one-way ANOVA: number of unperformed activities, and acuity indices 33

3.14 Plot of post-hoc analysis: effects of acuity indices on number of unperformed

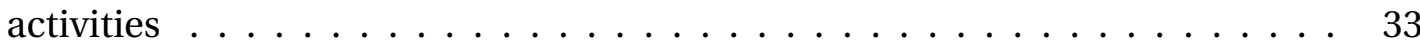




\section{List of Algorithms}

1 Transcript: nurse flowchart . . . . . . . . . . . . . . . . . 14

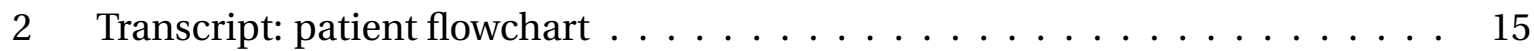




\title{
List of Abbreviations
}

\author{
ABM Agent-based Modelling \\ ANOVA Analysis of Variance \\ CAPCR Coordinated Approval Process for Clinical Research \\ CV Coefficient of Variation \\ CQI Continuous Quality Improvement \\ DES Discrete Event Simulation \\ ED Emergency Department \\ ER Emergency Room \\ GCRP Good Clinical Research Practice \\ GDP Gross Domestic Product \\ GPU Graphics Processing Unit \\ GRASP Grace Reynolds Application of the Study of PETO \\ HPPD Hours Per Patient Day \\ JIT Just-in-Time \\ LCG Linear Congruential Generator \\ MAE Maximum Acceptable Effort \\ MSD Musculoskeletal Disorder \\ MVC Maximum Voluntary Contraction \\ PETO Poland, England, Thornton, and Owens
}




$\begin{array}{ll}\text { RAM } & \text { Random Access Memory } \\ \text { REB } & \text { Research Ethics Board } \\ \text { RFID } & \text { Radio Frequency Identification } \\ \text { RN } & \text { Registered Nurse } \\ \text { RPN } & \text { Registered Practical Nurse } \\ \text { RQ } & \text { Research Question } \\ \text { SD } & \text { Standard Deviation } \\ \text { TQM } & \text { Total Quality Management }\end{array}$




\section{Chapter 1}

\section{Introduction}

The need for and use of Agent-based Modelling in healthcare are described in this chapter including an overview of research gaps in this field of study. The chapter ends with the presentation of the research questions for this investigation.

\subsection{Background}

Healthcare systems and strategies for their improvement have been the source of continued debates, dramatic investments, and a broad range of academic research. Healthcare systems consume a substantial percentage of the nation's income. In 2015, the healthcare expenditure in Canada was $10.5 \%$ of Gross Domestic Product (GDP), and in the United States it was nearly 17.1\% (IBRD 2015, Shah 2003). The goal of many countries is the continued enhancement of the quality of patient care and the improved efficiency of caregivers while decreasing costs (Collins \& Noble 1992, Enthoven \& Tollen 2005). This topic has been the object of significant research and theorization (Chaudhry et al. 2006).

Engineering disciplines, rooted in academia, have been utilized to achieve these aims. Methods such as Total Quality Management (TQM) and Continuous Quality Improvement 
(CQI) have been implemented successfully because the service level of healthcare systems can be considered a quality issue (Andersson 2007, Dahlgaard et al. 2011). For instance, Weiner et al. (2006) have studied 1784 hospitals in the United States that have implemented quality management approaches such as TQM and CQI since the 1990s and concluded that these implementations have had a significant positive effect on the quality of patient care; however, the success of implementing these management approaches is strongly dependent to the involvement of staff and also the fact that the healthcare system, as a service system, handle human issues and not goods.

Nevertheless, engineering systems are inclined to ignore the human element in system design, and this has led to high costs, poor quality, delays, and flaws in general system efficiency as well as deterioration in the health of employees such as Musculoskeletal Disorder (MSD) and burnout (Dul \& Neumann 2006, Neumann et al. 2009, Neumann \& Dul 2010). Burnout is a common issue among healthcare staff and related professions due to unmanaged stress and overwork (Gingras et al. 2010). For instance, the quality of the work environment of caregivers exerts a direct influence on their job satisfaction and on the quality of care they give to patients (Purdy et al. 2010). Issues such as weariness with working conditions and high workloads have led to injuries and even cognitive lapses in judgment. These can include errors of omission, e.g. failure to perform the correct activity such as not ambulating the patient per his/her needs (Harvey et al. 2015) or high workload management, which has even caused nurses to leave the profession (Purdy 2011).

Kalisch et al. (2009), Yoder (2010), and Yuan et al. (2011) investigated the phenomenon of ignoring human factors in the design of a system within healthcare. They determined that disregarding human factors has a counter-effect on desired aims such as productivity, efficiency, and cost, and can cause resistance to new system thinking and managerial alterations by increasing the occurrences of information-handling or motivational problems and gradual shift from focus on evidence based practice of employees to other managerial aspects such 
as short-term cost efficiencies (Karsh et al. 2006). By studying more than 150 hospitals in the United States, Aiken et al. (2008) found a strong relationship between the quality of nurses' work environment and size of their workload with the quality of care provided to patients. Therefore, the aim of a healthcare system design using a more pragmatic approach is to increase the level of quality and the efficiency of healthcare units without causing side effects to the health of personnel, as shown in Figure 1.1.

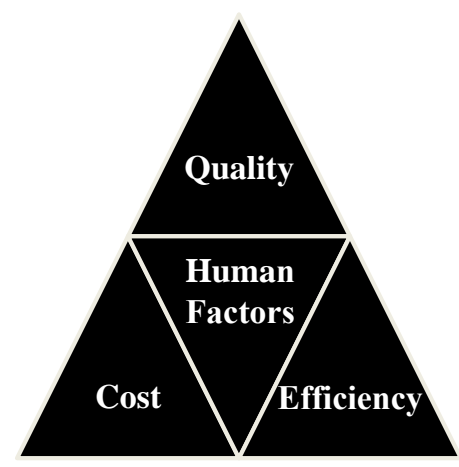

Figure 1.1. Aims in alteration of healthcare units and organizations

The definition of "human factors" used in this investigation is from the International Ergonomics Association (2015). Human factors is "the scientific discipline concerned with the understanding of interactions among humans and other elements of a system, and the profession that applies theory, principles, data and methods to design in order to optimize human well-being and overall system performance" or simply "fitting the task to the human" (Grandjean \& Kroemer 1997). By enhancing the human factors, including the work environment, these side effects such as resistance to a new system or burnout can be mitigated while quality of service to customers can be improved (Dul \& Neumann 2009, Neumann \& Dul 2010). However, implementing changes based on trial and error can be very expensive, and even hazardous, for organizations such as hospitals or clinics (Gaba 2004, Günal \& Pidd 2010). To avoid expensive renovations to the work environment and to decrease the danger of bad decisions, computerized simulations have been commonly used as a preliminary step for alterations to sensitive systems (Banks 2010). The computerized 
simulation and analytical studies are reinforcing and supporting each other in academic researches. As Banks (2010) stated computerized simulation is a more appropriate method: (a) when addressing interactions of a complex system such as factory or hospital with several internal types of entities, and uncertainties; $(b)$ organizational and work environmental changes, and effects of these alteration on the model's behavior; and (c) to observe how variables interact.

Simulations have mostly been done using the Discrete Event Simulation (DES) approach, a computerized simulation method based on the assumption that events and state variables occur only in a well-defined discrete set of moments in time (Karnon 2003, Swisher \& Jacobson 2002). As Günal \& Pidd (2010) elaborated, producing virtual but verified and validated models via DES allows engineers and managers to optimize the best solutions with regard to healthcare efficiencies, e.g. improving patient flow or cost efficient staff scheduling.

There have been studies performed on the utilization of DES for the application of human factors in a system. For example, DES has been used as a tool to better comprehend workplace health and identify possible injury levels in the early stages of the design of healthcare systems such as emergency systems (Duguay \& Chetouane 2007). The quest for better approaches in computerized simulations continues with an emphasis on how human factors can be used to evaluate and predict working conditions in work system design and therefore avoid issues that could arise after systems have been built.

According to Charfeddine \& Montreuil (2008), a new trend exists to model healthcare environments using Agent-based Modelling (ABM), as complex human-centred representations, instead of the traditional DES methodology. ABM is a novel computerized simulation approach based on a collection of autonomous decision-making objects called agents, which individually assess their situations and make decisions rooted in given rules (Borshchev 2013, Cimete et al. 2003).

Human-related attributes for entities such as the high level of individualization, com- 
plexity of activities and scenarios, and emphasis on interaction of agents have given the edge to ABM in healthcare analysis over other techniques like DES for characteristics such as model focus or run time (see Table 1.1 for a list of differences between DES and ABM) (Sibbel \& Urban 2001, Siebers et al. 2010).

\begin{tabular}{c|cc}
\hline Characteristics & DES & ABM \\
\hline Model focus & system & individual \\
Time domain & discrete & discrete \\
Run time & fast & slow \\
Rationalize decision making & not-applicable & applicable \\
Memoryless & applicable & not-applicable \\
\hline
\end{tabular}

Table 1.1. Prominent characteristics of ABM in comparison to DES (Bonabeau 2002, Barnes et al. 2013, Borshchev 2013)

ABM has its roots in Game Theory. Game theory, originally developed for economic studies, has given agents (entities) the ability to evolve from interaction with each other (Bonabeau 2002, Macy \& Willer 2002, Tesfatsion 2003, van Vuuren et al. 2012) by optimizing mathematical models of conflict and cooperation between intelligent rational decisionmakers (Varoufakis \& Hargreaves-Heap 2004). This premise is significant considering that many computerized simulation studies are intended to investigate issues with underlying elements in Queue Theory, which is the foundation of DES (Cassandras \& Lafortune 2009). It is essential to select the appropriate modelling method based on the specifications and eccentricities of the case since DES and ABM are not interchangeable with each other. Compared to DES studies and echoed by the fact that ABM is a new approach, ABM suffers from gaps, especially in the study of relationships between human factors and engineering design. ABM focuses solely on engineering or business perspectives and has not considered the health and well-being of employees. An example of ABM in emergency department analysis is shown in Figure 1.2 which was developed to study the relationship between the number of doctors on the call and waiting time for patients.

In recent decades, hospitals have gone through substantial modifications and have 


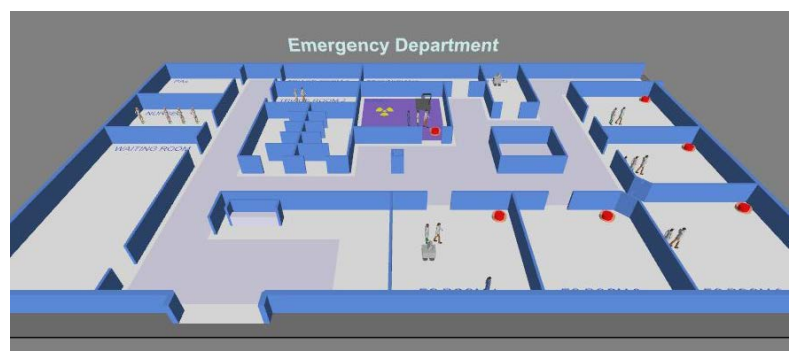

Figure 1.2. An example of ABM (AnyLogic 2015)

tended to downsize the nursing workforce, even to insufficient levels, due to the large cost of employing nurses (Pan et al. 2007). Nurses' intense physical and mental work, experienced in many other professions as well, negatively impacts the opportunity for recovery time, i.e. the period of time without accumulating fatigue (Rose et al. 2011) or period of time without stressors such as "meeting patients' needs, self-expectations, quantitative workload, and colleagues' inexperience" for nurses (Hall 2004, Yoder 2010). Recovery time is an essential part of the caregivers' work routine and can impact the success of work environment alterations. Considering the many occasions upon which nurses have to walk between different rooms even after care has been completed, the period of time spent walking per shift by nurses should not be neglected in any engineering design or alteration of healthcare units. Moreover, it should be noted that the sudden downsizing of staff, though cost efficient, can cause the caregivers to try to compensate for the care delivery delays to patients by extra effort, which often concludes with side effects such as residual fatigue and burnout (Cimete et al. 2003, Laschinger et al. 2001).

By successfully developing a virtual unit based on empirical evidence, it can be feasible to manipulate certain variables to test the possible impact of changes to the work and work environment of nurses in a simulated environment. In the future, the results could provide direction for studies on the well-being of nurses that can help them deliver high quality care. The very first step is to investigate the feasibility of Agent-based Modelling technology that could be used to test hypothetical changes in design to determine the best outcomes in two 
main categories: human factors of caregivers and quality of care to patients. Quality of care is considered a multidimensional concept and has several definitions in different academic disciplines (Wilde et al. 1994). For this investigation, quality of care is considered as the on-time delivery of care to patients, or the minimizing of the number of missed care tasks, i.e. activities that are not completed by nurses. For instance, Kalisch et al. (2009) has studied a sample of 459 nurses and concluded that more than $70 \%$ of nurses had nearly $44 \%$ rate of missed care in their workplaces.

By conducting a literature review that focuses on the use of ABM in the healthcare system, human factors, quality of care to patients, and staff workload, three main gaps have been identified as demonstrated in Table 1.2: (a) emphasising only emergency departments; (b) ignoring nurse/patient ratios; and $(c)$ ignoring the patient acuity index. Each gap is described in more detail in the next subsections.

\begin{tabular}{|c|c|c|c|c|c|c|}
\hline \multirow[t]{2}{*}{ Researches } & \multicolumn{2}{|c|}{ Unit Type } & \multicolumn{2}{|c|}{ Staff Ratio } & \multicolumn{2}{|c|}{ Severity of Illness } \\
\hline & Inpatient & Outpatient & Doctor/Patient Ratio & Nurse/Patient Ratio & Triage & Acuity \\
\hline Barnes et al. (2013) & No & Yes & Yes & No & Yes & No \\
\hline Charfeddine \& Montreuil (2008) & No & Yes & Yes & No & Yes & No \\
\hline Hongqiao et al. (2009) & No & Yes & Yes & No & No & No \\
\hline Laskowski \& Mukhi (2009) & No & Yes & Yes & No & Yes & No \\
\hline Taboada et al (2011) & No & Yes & No & No & Yes & No \\
\hline
\end{tabular}

Table 1.2. Current trends and found gaps in ABM and Healthcare studies

\subsubsection{Emphasis on Emergen'cy Departments}

Much of the research using ABM has been focused on the enhancement of the quality of care within outpatient settings such as the Emergency Department (ED). In these studies, the patient arrival or dismissal rate was already determined, either by using an exponential distribution or by modelling it with a continuous time domain simulation approach. The movements of patients in the emergency clinic were patterned with tools such as Radio Frequency Identification (RFID). ABM studies such as Barnes et al. (2013), Charfeddine \& Montreuil (2008), or Laskowski \& Mukhi (2009) focused on outpatient settings and inpatient 
settings are rarely taken into consideration. This investigation shifted its focus to inpatient units to address this inadequacy in academic studies.

\subsubsection{Ignoring Nurse/Patient Ratios}

Downsizing is a sensitive and important factor in contemporary healthcare systems. The reduction of staff is done mostly on the basis of cost efficiency, and human factors are rarely taken into consideration. Previous ABM studies which were focused on the productivity of healthcare systems concentrated on the number of doctors (Barnes et al. 2013, Hongqiao et al. 2009, Laskowski \& Mukhi 2009). Nursing work has not been addressed despite the fact that over $75 \%$ of patient care is delivered by nurses (Nursing Task Force 1999). Many non-ABM studies exist that clearly address the impact of nurse/patient ratios on the quality of patient care and the health and well-being of nurses such as length of waiting time for patients or stressors due to high rate of workload for nurses (Aiken et al. 2002, Needleman et al. 2002, Yoder 2010). Nurse/patient ratios refer to the number of patients assigned to a given nurse within a single shift, i.e. the larger the number of patients per nurse, the greater the nursing workload. By adding the nurse/patient ratios as an input variable to the experimental ABM design, this investigation has centred on the nurse as a vital caregiver. In this way, the study can examine the effects of nurse/patient ratios on aspects of workload such as percentage of time spent on the delivery of direct care, walking vs. recovery time, and number of unperformed care activities.

\subsubsection{Ignoring Patient Acuity Index}

In many ABM studies, there is a lack of awareness of the patients' acuity or severity of illness, as a factor influencing workload. Much ABM research studying the relationship between staffing and patients in clinics did not directly address patient acuity, such as the 
percentage of unstable patients in a clinic, or they considered all patients were homogeneous in terms of severity of illness and their associated care needs (Barnes et al. 2013, Stainsby et al. 2009, Taboada et al. 2011). Moreover, ABM studies that mentioned an acuity index merely considered it with triage in emergency rooms based on optimization of patients' waiting time in queue and not an indicator of average time spent with each patient during a specific period of time (Charfeddine \& Montreuil 2008, Laskowski \& Mukhi 2009). In practice, more severely ill patients would typically be admitted as inpatients, where acuity is an important and established factor in nurses' workload as mentioned in several studies of nurses' burnout (Cimiotti et al. 2012, Rafferty et al. 2007). By adding the patient acuity index to this investigation and thus increasing heterogeneity of agents (patients) in the virtual unit, the relationship between patient acuity and nursing workload is addressed.

\subsection{Research Questions}

The following research questions were addressed in this investigation:

RQ1 How can ABM be used to assess and predict nursing workload in an inpatient unit?

RQ2 How do nurse/patient ratios affect the quality of patient care and available recovery time for nurses in the virtual unit?

RQ3 How does change in patient acuity levels affect the quality of patient care and available recovery time for nurses in the virtual unit? 


\section{Chapter 2}

\section{Method}

The methods and approaches to address the research questions are elaborated upon in this chapter. The approach used for data collection is explained to show how the relevant metrics were acquired. The modelling was performed in two steps: first, creating and running the virtual unit with $\mathrm{ABM}$, and then adapting it using the results of the collected data.

\subsection{Study Design and Data Collection}

The data was collected and analyzed using quantitative methods to address issues such as common nursing care activities, effort level for identified nursing care activities, and acuity. An interview process was used to obtain subjective ideas on the potential of the current system for enhancement of care delivery.

\subsubsection{Sample and Setting}

Considering the exploratory nature of this technology development research, any inpatient unit at the designated hospital was considered acceptable for the case study of this investigation. The selected unit was located in a downtown area of a large urban centre in Ontario. 
The inpatient medical unit utilized twelve-hour shifts (day/night) and employed full- and part-time Registered Nurses (RNs) and Registered Practical Nurses (RPNs). The manager responsible for nurses' workload measurement, the GRASP manager, was interviewed about the GRASP metrics. For the convenience of the participant, the interview was held in the manager's office in the designated hospital.

\subsubsection{Gaining Access to Participants and Quantitative Data}

Gaining access was a preliminary and mandatory step in collecting the required data for the validation of the virtual unit. Initially, the individual responsible for nurses' workload management at the selected hospital was identified for the purpose of an interview. The manager was provided with a recruitment letter, a brochure, and a consent form. A convenient time for the interview was established via email. Two copies of the consent form were signed by the manager and one copy was given to the participant. Elaboration of the background and routines supporting the GRASP data was requested during the meeting and the anonymized GRASP data of the selected unit was received after the meeting.

Protection of Human Subjects - Ethical Considerations: Only the researcher had access to the collected data. Neither the participant's name nor the name of the hospital was mentioned. Only grouped data, such as activity durations, were reported. Hard copies of recorded data were stored in a locked cabinet at the workplace of the researcher. The digital data was stored on password-protected laptops and in encrypted storage clouds.

The Research Ethical Board (REB) of Ryerson University assessed the current investigation and determined that this study did not require REB approval due to its focus on process evaluation and improvement. Nonetheless, the research quality integration department of the designated hospital mandated that all researchers had to pass the Good Clinical Research Practice (GCRP) test to ensure they understood the rights of participants and maintained 
data integrity. Later, the REB of the designated hospital assessed this investigation by reviewing the Coordinated Approval Process for Clinical Research (CAPCR), provided by the researcher, and authorized the data collection.

\subsubsection{Interviews with the GRASP Manager}

A one-hour meeting with the GRASP manager was held during the data collection period. The aim of the meeting was to discuss and comprehend the system used to capture nurses' workload and to request the GRASP data of the selected unit. During the meeting, questions were addressed to clarify the premise behind GRASP data in general and the accuracy of GRASP data of the selected unit in particular. During the interview, clarifications were requested to better comprehend received quantitative data, such as clarifications on nomenclature and metric units, to help create valid inputs for the virtual unit.

The investigation used GRASP metrics. Grace Reynolds Application and Study of PETO (GRASP) data is a standardized and validated metric system for the analysis of nursing care provided to patients (Farrington et al. 2000). It was first developed and used in Grace Hospital, North Carolina, by a group of nurses nicknamed PETO (Poland, England, Thornton, and Owens), in the 1970s (Slee et al. 2001, Lee 2008). GRASP data is an accepted and validated measurement system for determining workload and productivity of nurses and has been used by hospitals for decades to report on nursing workload to governments, such as Ontario Ministry of Health and Long-Term Care.

GRASP data is based on two factors: duration and frequency of interventions, i.e. the external manifestations of the internal process that nurses perform in order to provide care and meet patients' needs (GRASP Systems International 2012). At the end of a shift, a nurse enters the number of his/her interactions with patients for each activity using standardized criteria. By analysis of GRASP data of the selected medical unit, i.e. durations and frequencies of nursing care activities per day shift for a period of one month, it was possible to estimate 
necessary the weighted arithmetic average for each activity as summarized in Section 2.3. GRASP data from one nurse was acquired. The GRASP data of the selected unit had the following characteristics (GRASP Systems International 2012): (a) The GRASP data had an adjusted time of $15 \%$ to reflect unpredictable care or care not listed in the GRASP data, i.e. all durations were divided by 0.85 ; and $(b)$ The GRASP manager provided a value of $60 \%$ for the accuracy of GRASP data of the available unit, for this investigation due to the weak compliance level in reporting nursing workload. This inaccuracy was treated as a limitation in data mining (see Chapter 4). All durations have an additional allowance for fatigue (10\%).

\subsection{Modelling}

\subsubsection{ABM Software Selection}

Few simulation software products can produce ABM virtual environments. NetLogo and AnyLogic are the prominent products in the industrial and academic sectors. AnyLogic, though new in comparison to NetLogo (which is free), was selected for this investigation due to three desirable factors. In comparison with NetLogo which utilizes its own programming language, AnyLogic uses the powerful object-oriented language, JAVA. Hybrid methods (combinations of different simulation approaches) are possible in AnyLogic. Moreover, AnyLogic has the capacity to produce three-dimensional output. For this investigation, AnyLogic version 7.2 was used.

\subsubsection{Model Conceptualization}

Initially, the shell of the virtual unit was created and prepared to receive empirical data. After consultation with an experienced nurse and academic researcher, a preliminary set of data (activities and their durations) was estimated and used to create the virtual unit. The 
created virtual unit was presented to the Director of Practice-Based Research \& Innovation and the Clinical Research Manager for further evaluation and approval of the feasibility of the created virtual unit. After the analysis of the GRASP data, modifications to the model were initiated using actual workload data from the inpatient unit.

\section{Flowcharts for Agents}

As with all other computerized simulations, entities (agents) require clear algorithms of movements and possible events, called a flowchart (Tsuji et al. 1993). ABM requires rules of interaction for each type of agent explicitly. Flowcharts of nurses and patients (agents in the virtual unit) illustrate how nurses in the virtual unit interact with patients to deliver care, and how patients are received and initiated into the virtual unit. Nurses can also enter/exit the virtual unit during the simulation per researcher instruction (see Algorithm 1, Algorithm 2, Figure 2.1, and Figure 2.2). The pseudocodes in JAVA programming language are presented in Appendix A.

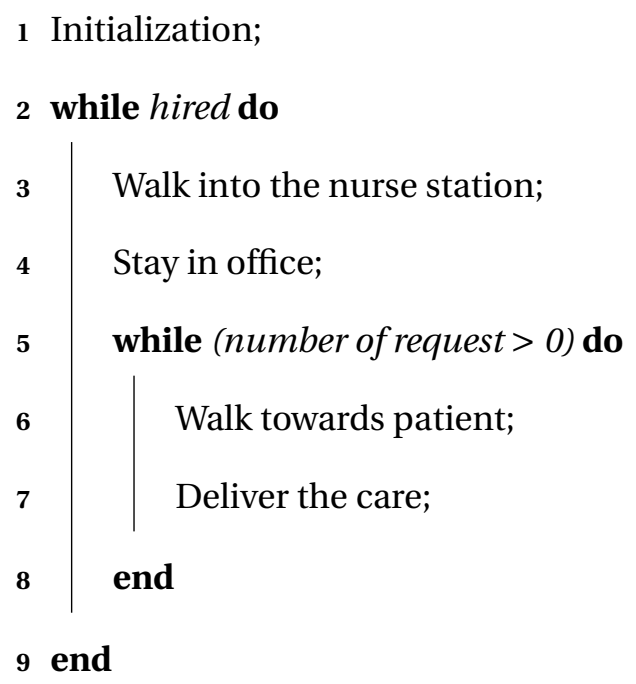

Algorithm 1: Transcript: nurse flowchart (Borshchev 2013) 


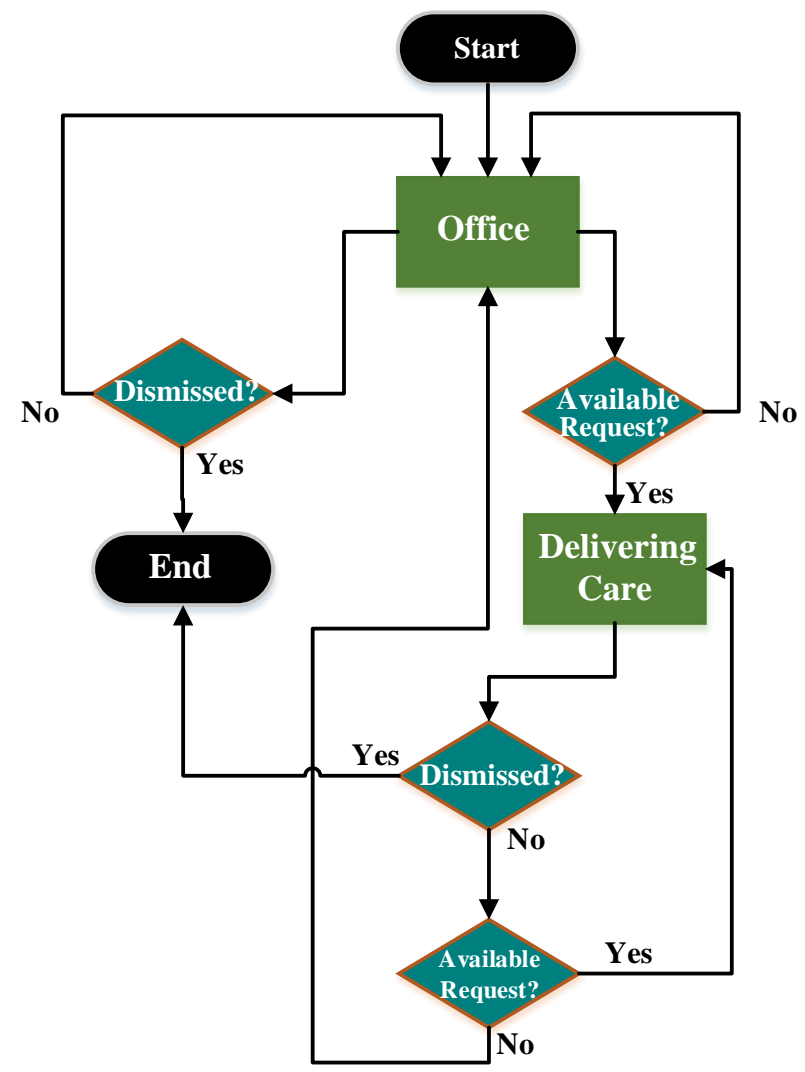

Figure 2.1. Flowchart of nurses in the virtual unit (Borshchev 2013)

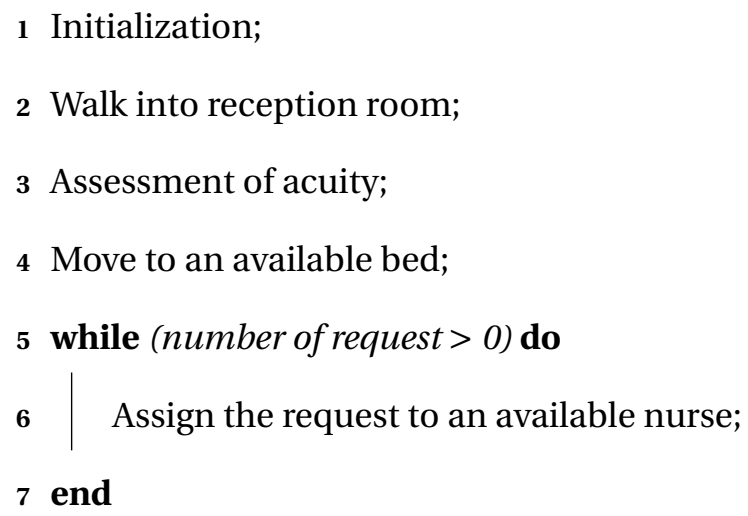

Algorithm 2: Transcript: patient flowchart

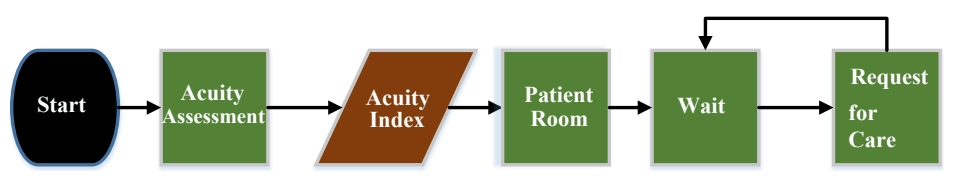

Figure 2.2. Flowchart of patients in the virtual unit 


\section{Layout of the Unit}

The layout of a possible virtual clinic was estimated since access to the blueprint of the selected clinical unit was not provided. The layout consisted of fifteen rooms and thirty beds (each room had two beds), and rooms were separated by walls and were not connected to each other. The dimensions of the patient rooms were approximately $10 \times 5$ metres, which is consistent with the average size of inpatient rooms at Clarian West Medical Center (Indiana), Parker Adventist Hospital (Colorado), and St. Rose Dominican Hospital (Nevada) (Harvey et al. 2006). A medication room and a patient/visitor's lounge for patients were located in the west wing of the unit. The nurses' station was located in the middle of unit. The nursing station had a capacity of two to eight nurses (agents). Two walk paths were considered for agents: (a) Patients' path: the path that patients take to their rooms from the reception room at the initial stage of simulation run; and $(b)$ Nurses' walk path: the path that nurses use to walk back and forth between different rooms and their station.

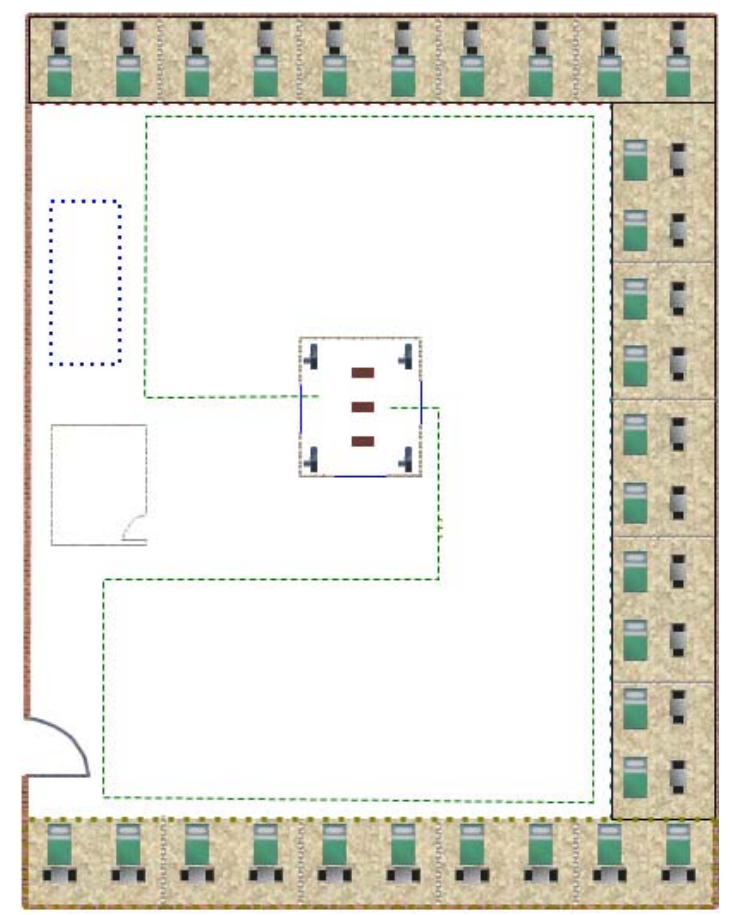

Figure 2.3. Layout of the virtual unit 


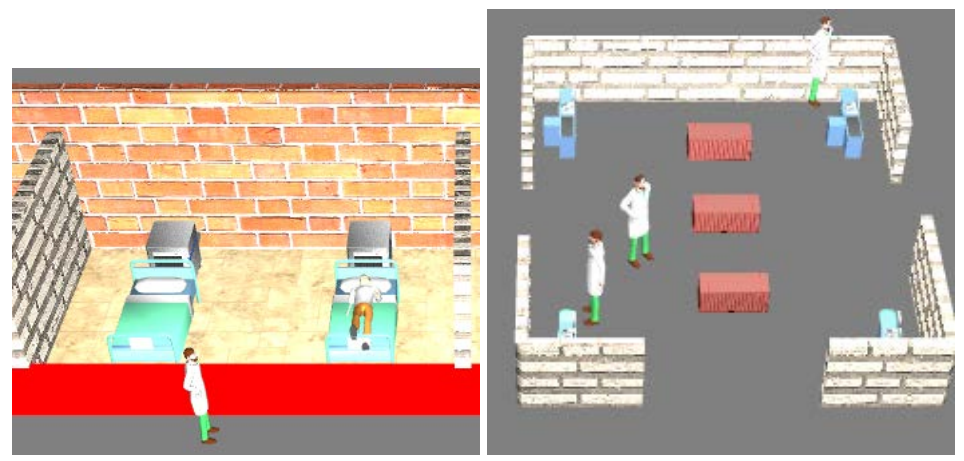

Figure 2.4. Details of rooms and nurse station

\section{Activities}

In this investigation, the most common activities for nurses were included, as shown in Figure 2.5 and Table 2.1. Elimination, hygiene, medication, non-patient activities, treatments, vascular access, and vital signs were considered, through analysis of GRASP data.

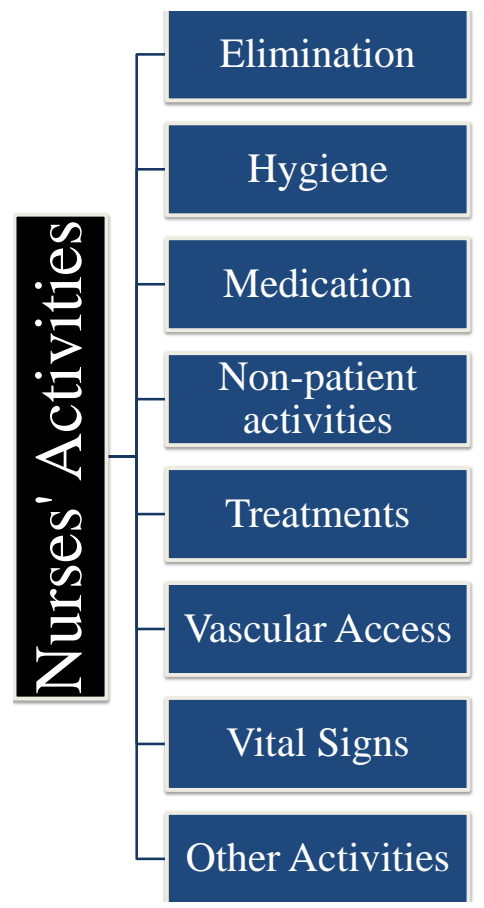

Figure 2.5. Considered activities for nurses 


\begin{tabular}{l||l}
\multicolumn{1}{l||}{ Activity } & \multicolumn{1}{c}{ Description } \\
\hline \hline Elimination & $\begin{array}{l}\text { to help a conscious/unconscious patient with } \\
\text { toileting }\end{array}$ \\
\hline Hygiene & to clean a conscious/unconscious patient \\
\hline Medication & to prepare and administer medications \\
\hline Non-patient activities & $\begin{array}{l}\text { activities that are only indirectly related to pa- } \\
\text { tient care such as documentation/paperwork }\end{array}$ \\
\hline Other activities & $\begin{array}{l}\text { All other activities related to patient care (e.g. } \\
\text { after death care) }\end{array}$ \\
\hline Treatments & $\begin{array}{l}\text { general term for treatments such as wound } \\
\text { dressings or chest tube removal }\end{array}$ \\
\hline Vascular Access & to draw a blood sample for diagnostic purposes \\
\hline Vital Signs & to measure vital signs
\end{tabular}

Table 2.1. List of activities and their descriptions in the selected unit

\section{Independent Variables}

Two independent variables were used in this investigation: (a) Nurse/patient ratio; and (b) Acuity indices of patients.

Nurse/Patient Ratio The number of patients assigned to a single nurse. This addressed the tendency to increase the staff ratio in order to reduce care costs, i.e. increase the number of patients per nurse (see Chapter 1). Three staff ratios were considered: Low (L), one nurse to four patients; Medium (M), one nurse to five patients; High $(\mathrm{H})$, one nurse to six patients. These ratios are consistent with nurse/patient ratios of inpatient units at Clarian West Medical Center (Indiana), Parker Adventist Hospital (Colorado), and St. Rose Dominican Hospital (Nevada) respectively (Harvey et al. 2006) also consistent with number of patients per nurse in studies such as Aiken et al. (2002), Lang et al. (2004).

Acuity Indices of Patients This study divided the patients' acuity indices into three levels assuming that frequency of nursing care activities and thereby workload would increase with each subsequent acuity level. For a description of how acuity levels were calculated, refer to 
the discussion provided in section 2.5 .

This study divided the patients' acuity indices into three levels:

Acuity Index One reflected stable patients. In the default mode, $25 \%$ of patients in the virtual model belong to this category.

Acuity Index Two was assigned to patients with a medium level of illness. In the default mode, $50 \%$ of patients belong to this category.

Acuity Index Three referred to unstable patients. In the default mode, $25 \%$ of patients in the virtual model belong to this category.

\section{Dependent Variables}

Two dependent variables were used in this investigation: (a) Recovery time: Three states were assumed for nurses including recovery time, walking, and delivering the care to patients. By altering the defined independent variables, the researcher could observe how they affected the available recovery time of nurses; and $(b)$ Average number of unperformed activities in a shift: measurement of quality of care in this research was the average number of activities waiting (in queue) to be performed by the relevant nurse in a shift. This dependent variable is consistent with the definition of quality of care in Chapter 1, and the researcher could observe the results of nurses' decisions as they were impacted by staff ratio and acuity indices.

\subsection{Data Analysis Plan}

A data analysis plan is a preliminary step in model translation that includes the act of implanting the model in computer code, including timing and general procedures and the translations of the conceptual models into computer simulation program representations 
(Rossetti 2010). In this investigation, data analysis was performed by data mining, the use of an experimental design to answer RQ2 and RQ3, and also ongoing verification and validation of the virtual unit throughout the investigation.

\subsection{Verification and Validation}

Schlesinger (1989) defined the verification and validation in the content of computerized simulation respectively: (a) ensuring that the computerized model and its implementation are correct; and $(b)$ ensuring that the computerized model within its domain of applicability possesses a satisfactory range of accuracy consistent with the intended application. Verification and validation were performed on an ongoing basis throughout the investigation. First, the data mining was based on GRASP data. Flowcharts for each agent were developed, and several tools were designed for output analysis as described below:

Charts (pie charts and column charts) were used to demonstrate the level of utilization of each agent at any moment of the simulation and also the percentage of time spent on walking, delivering care, and having recovery time. Results of different scenarios while the simulation was running were consistent with common sense assumptions, e.g. more staff means fewer unperformed activities. Results of several different scenarios, such as adding or removing patients or nurses, were checked during the running of the simulation, and they were consistent. Two external managers from the selected hospital observed the model, commented on the level of progress, and provided a face validity check for the approach of the virtual unit. Animation was created to demonstrate the movement and interaction of agents. Outputs are analyzed in Chapter 4 and have been compared with academic literature, showing similar effects. Several iterations were performed for each output. Each replication was unique and randomly generated.

AnyLogic software has an optimization toolbox, OptQuest, which was not available for 
this investigation. Nevertheless, the verification of outputs was performed in two steps: (a) Different seed, initial start value in a simulation run, was used for each replication; and (b) Ten runs were performed for each test and the average value was found and used (see Appendix B).

The Linear Congruential Generator (LCG) was used to verify the randomness of the model because it is a well-defined, general purpose random number generator (Banks 2010) and also a standard random number generator (48 bits seed) in the JAVA programming language (Borshchev 2013), where $X$ is the sequence of pseudorandom number, $m$ is the modulus, $a$ is the multiplier, and $c$ is the increment.

$$
\begin{gathered}
X_{n+1}=\left(a X_{n}+c\right) \quad \bmod \quad m \\
m=2^{48}, \quad a=5 \text { DEECE } 66 D_{16}, \quad c=11
\end{gathered}
$$

For estimating the number of necessary replications, the Bank's (2010) method is used; where $\varepsilon$ stands for level accuracy and $\alpha$ for confidence interval level.

$$
\begin{gathered}
\text { Initial number of Runs } \geq\left(\frac{Z_{\alpha / 2} \times S}{\varepsilon}\right)^{2} \\
\text { Needed Number of Runs } \geq\left(\frac{t_{\alpha / 2, R^{\prime}-1} \times S}{\varepsilon}\right)^{2}
\end{gathered}
$$

The results of calculations and analysis of Equation (2.2) and Equation (2.3) are presented in Appendix B. 


\subsection{Data Mining of GRASP Data}

Data mining is defined as the procedure by which analysts apply the tools of mathematics and statistical testing to historical data in order to identify relationships, patterns, or affiliations among variables or sections of variables in the data to gain a greater insight into the underpinnings of the processes (Kudyba 2001). After analyzing the received GRASP data, the metrics of the selected unit were sorted and cleansed: (a) Activities without name tags or frequencies were removed (cleansing); (b) Only day shifts were considered (cleansing); and (c) Activities and their frequencies were arranged per each patient (sorting).

\begin{tabular}{cccc}
\hline Activity & Sub-activity & Number of Intervention & Duration (min) \\
\hline Medication & Oral/Topical/GTTS/Puffers/Supp Meds & 2 & 18.02 \\
Vital Signs & TPR, BP including Oximetry & 3 & 6.76 \\
Vascular Access & IV Maintenance & 1 & 5.63 \\
Vascular Access & Blood Collection from Central/PICC Line & 1 & 5.63 \\
Nutrition & Feeds Self/Family Assist/NPO & 2 & 6.76 \\
Elimination & Toileting with Minimal Assistance & 2 & 11.26 \\
Treatments & Oxygen Therapy/Titration & 1 & 4.51 \\
\hline
\end{tabular}

Table 2.2. Example of GRASP data received from the selected unit

Later, it was understood that each activity (see Table 2.1) had its own set of sub-activities in GRASP data, e.g. one treatment includes several sub-activities such as chest tube removal or oxygen therapy with different durations (see Table 2.2). By using the weighted arithmetic mean method, the averages (means) of the activities were calculated (James 2006):

$$
\bar{X}=\frac{\sum_{i=1}^{k}\left(x_{i} \times v_{i}\right)}{n}
$$

Where $x_{i}$ and $v_{i}$ are the sub-activity duration and frequency respectively. Moreover, $n$ is:

$$
\sum_{i=1}^{k} v_{i}
$$


After analysis of GRASP data, the durations for each activity were calculated based on weighted arithmetic means (see Table 2.3).

\begin{tabular}{cc}
\hline Activity & Duration (min) \\
\hline Elimination & 24.9 \\
Hygiene & 10.1 \\
Medication & 23.3 \\
Non-direct Care & 56 \\
Other Direct Activities & 34.2 \\
Treatments & 13.6 \\
Vascular Access & 12.3 \\
Vital Signs & 24.7 \\
\hline
\end{tabular}

Table 2.3. Calculated durations (weighted arithmetic means) of considered activities

The GRASP data did not contain any information about acuity indices. To solve this issue, this investigation used the Hours Per Patient Day (HPPD) approach; reflecting the average number of nursing care hours required to care for a patient within a 24 hour period. HPPDs for a period of one month were grouped and sorted in ascending order to estimate the proportion of care received for each of three Acuity Indices using the following steps: (a) Cleanse the data; (b) Calculate the data based on HPPD, i.e. sum all the care a patient received per day; $(c)$ Sort the HPPDs and divide them into three equal groups; and $(d)$ Compare the value of median of the each group with the other two groups with assumption that the smallest median belongs to acuity index one. The proportions of frequency of care received for each of the three acuity indices $(\propto)$ were as follows: 1 for the acuity level of one, 3 for the acuity level of one, and for the acuity level of one 8.1.

\subsubsection{Experimental Design}

An experiment was defined with regard to RQ2 and RQ3, i.e. effects of staff ratio and acuity indices on quality of care and available recovery times for nurses. Two responses were determined based on the two dependent variables: number of unperformed activities and 
available recovery time (\%).

Considering that three levels of intensity (Low, Medium, and High) were selected for each factor-staff ratio and acuity, all combinations $(3 \times 3)$ were tested to create a comprehensive set of results. Each test was performed in ten replications. Confidence interval of $95 \%$ was selected for the purpose of analysis $(\alpha=5 \%)$.

\begin{tabular}{ccc}
\hline Test & Staff Ratio & Acuity \\
\hline$\# 1$ & High & High \\
$\# 2$ & High & Medium \\
$\# 3$ & High & Low \\
$\# 4$ & Medium & High \\
$\# 5$ & Medium & Medium \\
$\# 6$ & Medium & Low \\
$\# 7$ & Low & High \\
$\# 8$ & Low & Medium \\
$\# 9$ & Low & Low \\
\hline
\end{tabular}

Table 2.4. Matrix of nine defined tests

For further comprehension of differences in the outputs of the experiment, Analysis of Variances (ANOVA) was used. ANOVA is a statistical tool used to compare the averages of more than two sets of similar data in analysis (Bergman \& Klefsjö 2009, Montgomery 2012). Regarding each test (see Table 2.4), the results for each response were analyzed against each other to better comprehend the effects of factors on each response. By analysis of the posthoc tests of ANOVA results in this investigation, the effects of staff ratio and acuity indices on the defined responses were studied. For the post-hoc analysis (95\% confidence interval), this investigation selected the Tukey procedure due to its power in pairwise comparisons.

Null hypothesis All means are equal

Alternative hypothesis At least one mean is different

Significance level $\quad \alpha=0.05$

For performing statistical analysis, Minitab 17 and MATLAB 2015a were used. 


\section{Chapter 3}

\section{Results}

In this chapter, the results of the investigation of the three research questions are described in accordance with the methodology explained in the preceding chapter. The results are presented in descriptive and graphic styles.

\subsection{RQ1: ABM and the Virtual Unit}

In this investigation, metrics based on the defined research questions were gathered and analyzed by successfully creating a model based on assumed data and later adapting it to the collected data from the selected unit. The created virtual unit (see Figure 3.1) was based on GRASP data and adapted through interviews and data mining, as described in the preceding chapter.

The computerized model (or the virtual unit) was created with two changeable independent variables:

Staff Ratio The number of patients (twelve to thirty patients) and number of nurses (two to eight nurses) could be altered during the simulation run. This capability allowed the researcher to create and observe the results of different possible staff ratios. 

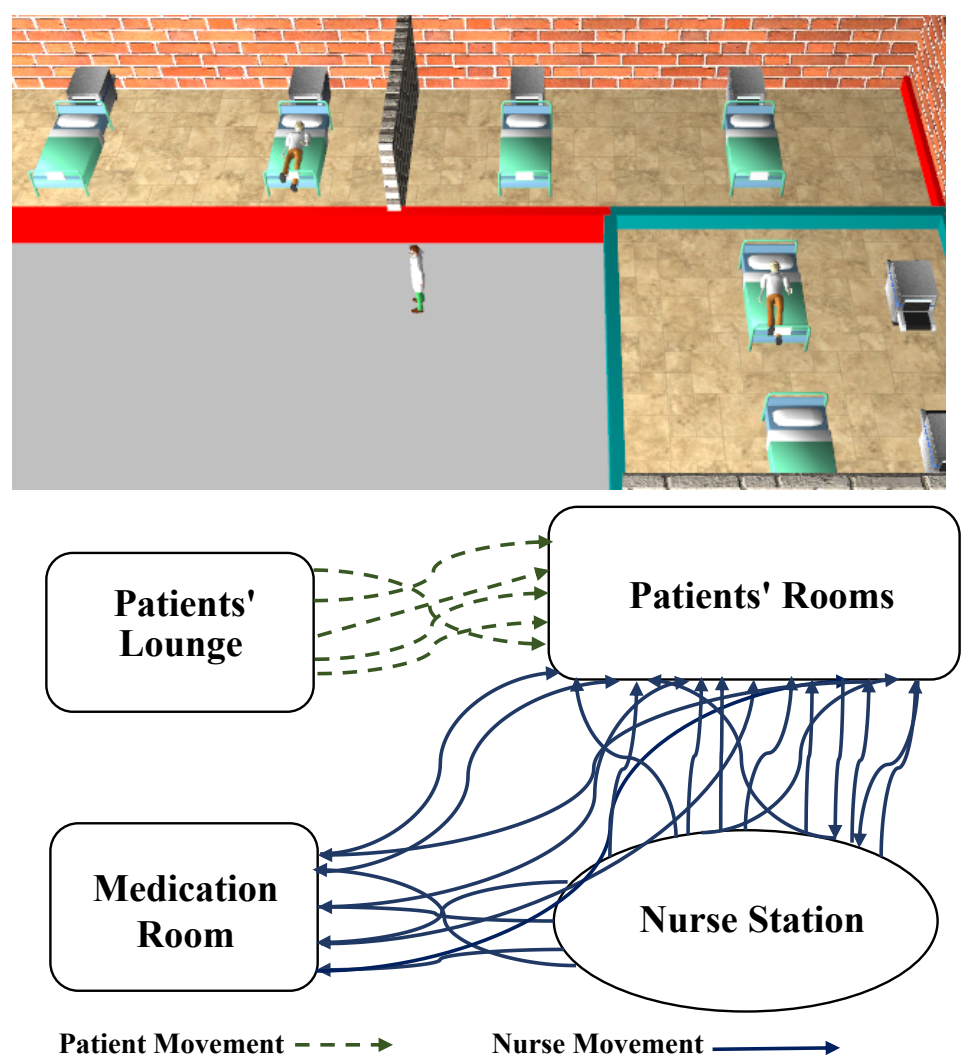

Figure 3.1. Example of movements of agents in the virtual unit: three-dimensional and spaghetti plots respectively

Acuity Index is the percentage of patients in each acuity index that could be changed to allow the researcher to observe the effects of this independent variable.

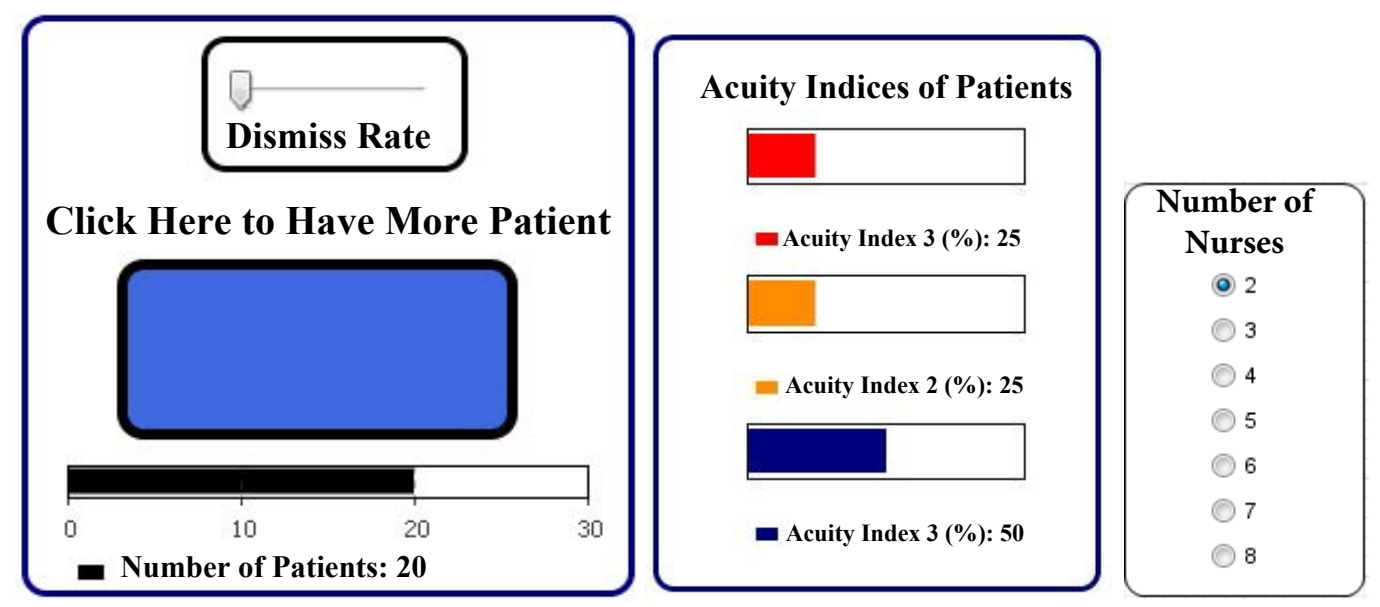

Figure 3.2. Tools developed for altering independent variables during computerized simulation 
By shifting the focus of computerized simulation metrics from accumulated results to moment-based metrics, the virtual unit allowed the researcher to observe the results of the alterations of independent variables directly. The model produced two primary output time-history charts: (a) Time-stack bar chart for the nurses' conditions such as walking or delivering care; and $(b)$ Time-series plot for the number of unperformed activities for patients. For instance, the results for the alterations of staff ratios are shown in Figure 3.3, which demonstrates the effects of staff ratio on the two dependent variables: available recovery time and number of unperformed activities.
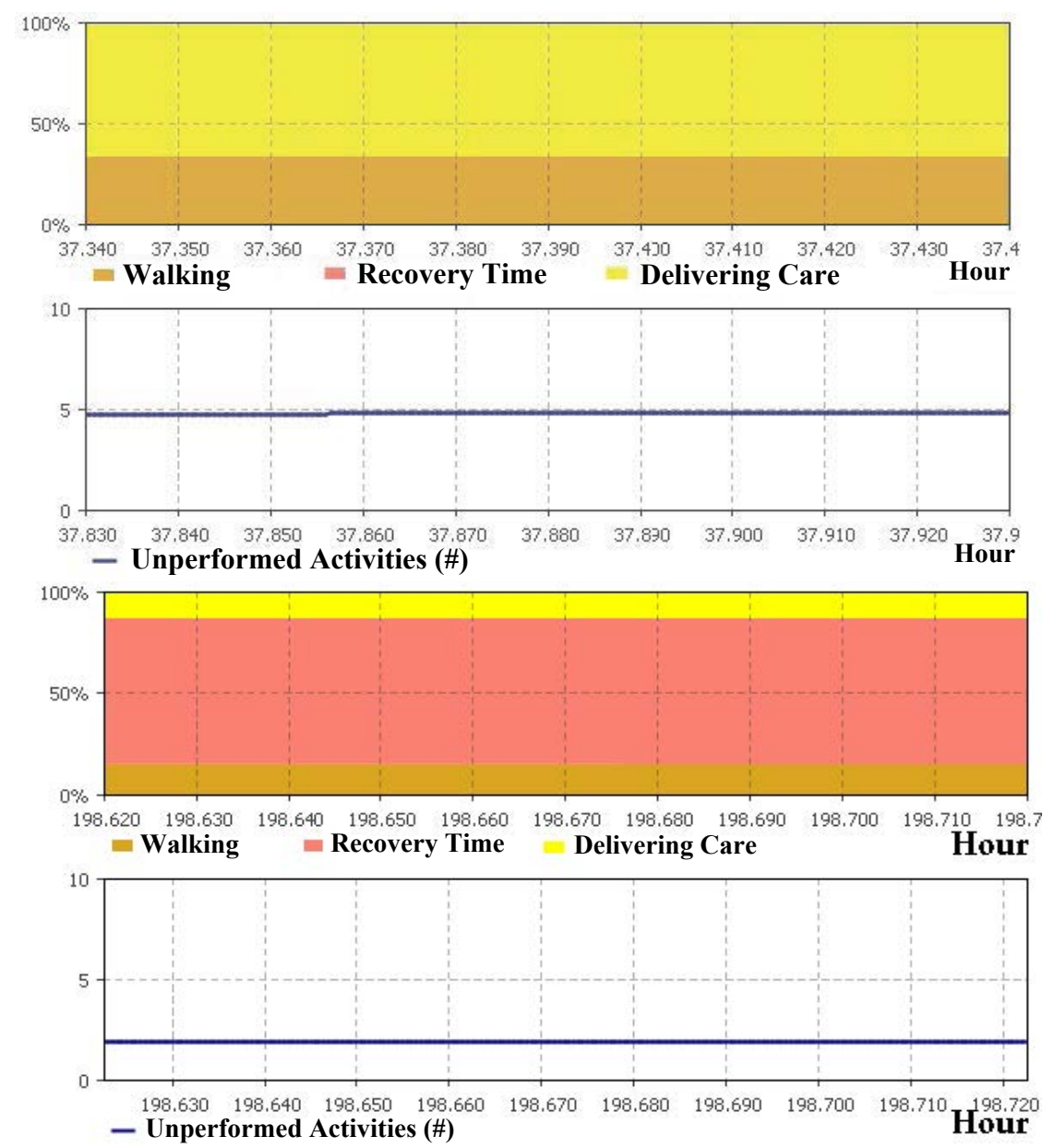

Figure 3.3. Time-stack and time plot charts for ratios of one nurse to nine patients (top) and one nurse to three patients (bottom)

The successful creation of the virtual unit as a prototype model for an inpatient unit by 
utilizing ABM created a customizable, adjustable, and expandable model. The model can be used as a benchmark in the development of new approaches in computerized simulation in human factors and quality assurance studies for inpatient units by the analysis of the relationship between staff ratio and patient acuity with available recovery time for caregivers (during or at the end of the shift) and number of missed cares simultaneously.

\subsection{Experimental Design Results}

Regarding the statistical experiment of the effects of staff ratio and acuity of patients on available recovery time and number of unperformed activities (described in Chapter 2), nine tests were done with ten replications for each test. Mean, Standard Deviation (SD), and Coefficient of Variation (CV) were calculated for each test and are shown in Table 3.1. As the staff ratio decreases (number of patients per nurse increases) and the acuity index population increases, the available recovery time decreases and the number of unperformed activities increases, as shown in Figure 3.4. The results were first analyzed graphically using box plots to better comprehend the nature of the collected outputs of the virtual unit and to understand the span of outputs through their standard deviations (Figure 3.5). Moreover, the error bars with 95\% confidence intervals are shown in Figure 3.6 for elaboration of the standard deviations of results of the nine tests listed in Table 3.1.

\begin{tabular}{|c|c|c|c|c|c|c|c|c|}
\hline & \multirow{2}{*}{\multicolumn{2}{|c|}{ Test }} & \multicolumn{3}{|c|}{ Recovery Time (\%) } & \multicolumn{3}{|c|}{ Unperformed Activities (\#) } \\
\hline & & & Mean & $\mathrm{SD}$ & $\mathrm{CV}$ & Mean & $\mathrm{SD}$ & $\mathrm{CV}$ \\
\hline$\# 1$ & High & High & 16.00 & 0.88 & 0.055 & 2.70 & 0.15 & 0.056 \\
\hline \#2 & High & Medium & 23.00 & 2.29 & 0.099 & 2.10 & 0.13 & 0.062 \\
\hline \#3 & High & $\mathrm{L}$ & 33.00 & 1.57 & 0.048 & 1.90 & 0.10 & 0.053 \\
\hline \#4 & Medium & High & 38.00 & 2.20 & 0.058 & 2.20 & 0.10 & 0.045 \\
\hline \#5 & Medium & Medium & 43.00 & 2.81 & 0.065 & 1.80 & 0.07 & 0.039 \\
\hline \#6 & Medium & Low & 47.00 & 2.80 & 0.060 & 1.60 & 0.30 & 0.188 \\
\hline \#7 & Low & High & 44.00 & 2.70 & 0.061 & 1.90 & 0.19 & 0.100 \\
\hline \#8 & Low & Medium & 51.00 & 2.70 & 0.053 & 1.50 & 0.81 & 0.540 \\
\hline$\# 9$ & Low & Low & 59.00 & 3.00 & 0.051 & 1.30 & 0.90 & 0.692 \\
\hline
\end{tabular}

Table 3.1. Results of available recovery time (\%) and average number of unperformed activities for the ten replications of each condition 


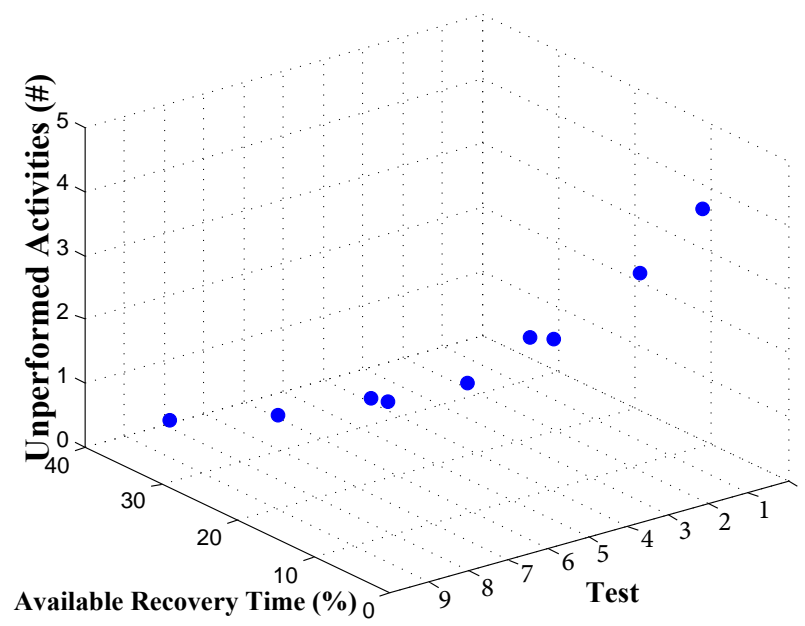

Figure 3.4. Three-dimensional plot of results of two responses: available recovery time and number of unperformed activities
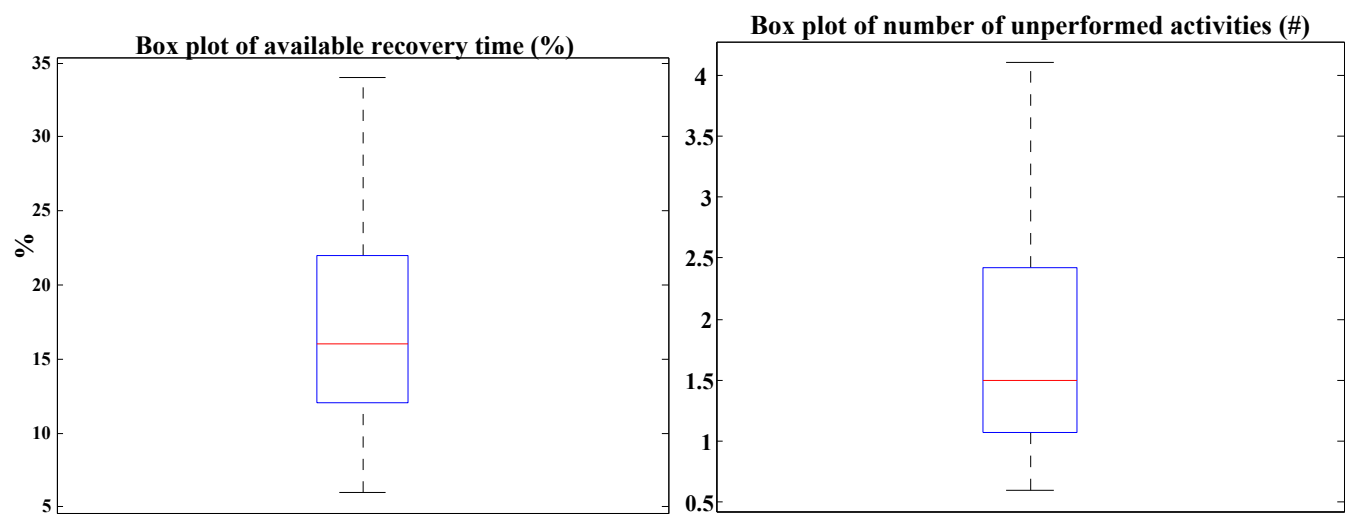

Figure 3.5. Box plots of results of two responses: available recovery time and number of unperformed activities respectively
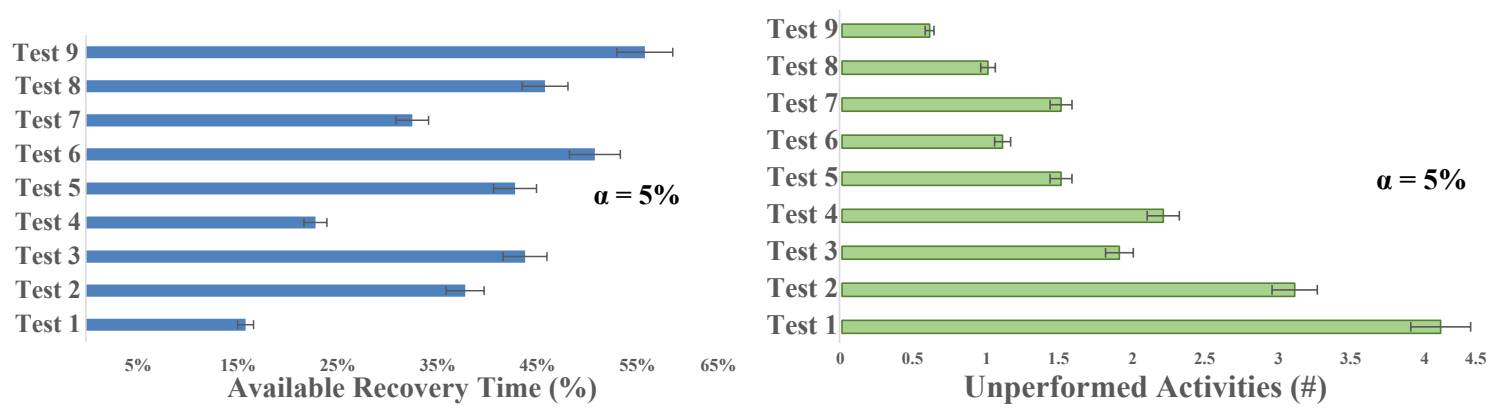

Figure 3.6. Bar charts with error bars ( $\alpha=5 \%)$ : available recovery time, and number of unperformed activities 


\subsection{RQ2: Effect of Staff Ratio}

\subsubsection{Effects on Available Recovery Time (\%)}

The results of ANOVA $(\mathrm{F}=5.47, \mathrm{p}=0.044)$ show that the $\mathrm{p}$-value is smaller than 0.05 , which demonstrates a noteworthy impact of changes in staff ratio. Therefore, it can be understood that staff ratio has a significant effect on available recovery time, as shown in Figure 3.7. Moreover, the result of the Tukey post-hoc analysis demonstrates that significant differences in available recovery time exist between staff ratio $(\mathrm{H})$ and staff ratio $(\mathrm{L})$ (see Figure 3.8).

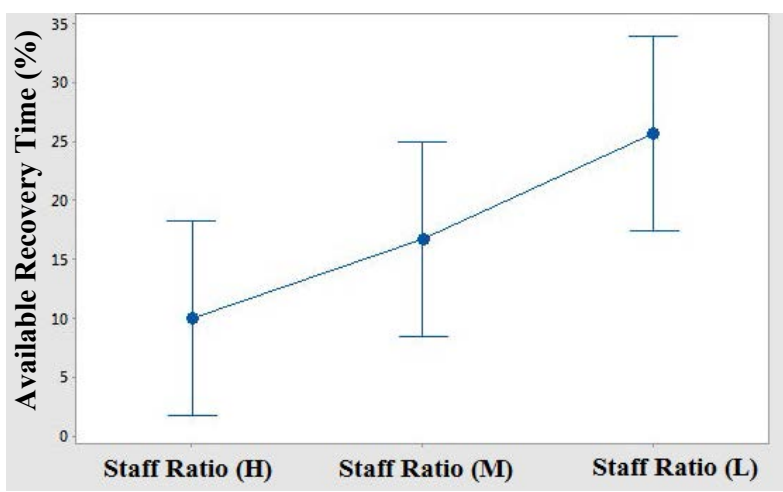

Figure 3.7. Plot of one-way ANOVA ( $\alpha=5 \%$ ): available recovery (response), and staff ratio

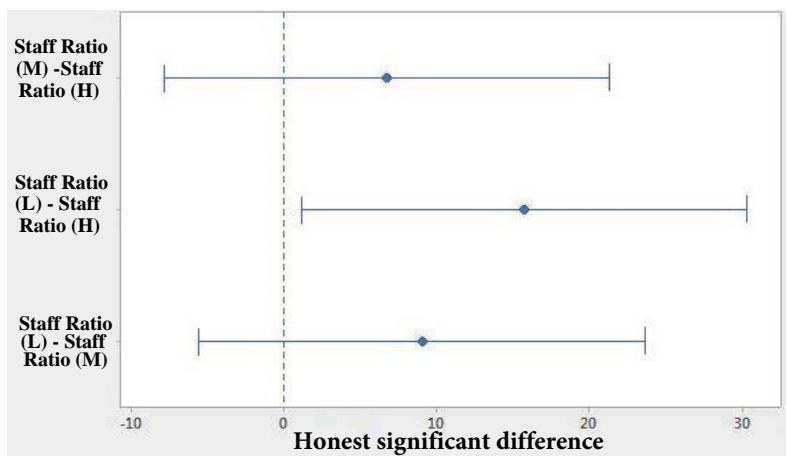

Figure 3.8. Plot of post-hoc analysis: effects of staff ratio on available recovery time 


\subsubsection{Effects on Number of Unperformed Activities}

The results of ANOVA $(\mathrm{F}=4.54, \mathrm{p}=0.043)$ show that the $\mathrm{p}$-value is smaller than 0.05 . Therefore, staff ratio has a significant effect on the number of unperformed activities as shown in Figure 3.9. The result of the Tukey post-hoc analysis demonstrates that significant differences in the number of unperformed activities exist between staff ratio $(\mathrm{H})$ and staff ratio $(\mathrm{L})$. It can be observed that staff ratio has significant effects on both available recovery time and the number of unperformed activities (see Figure 3.10).

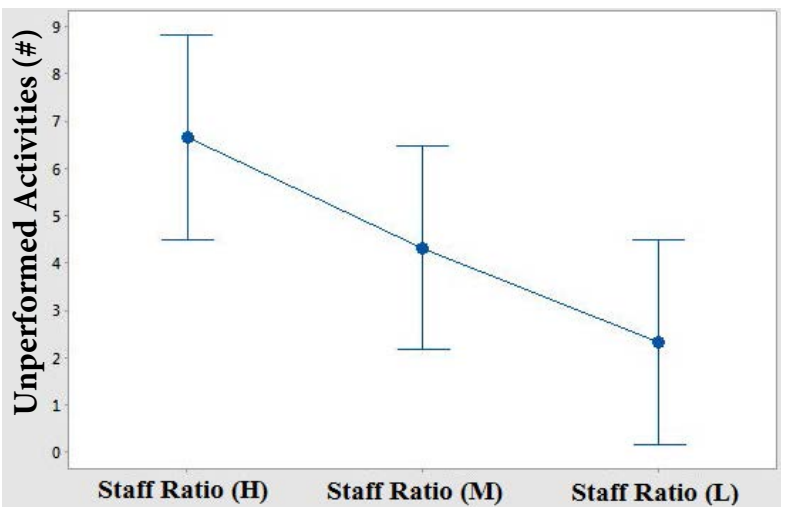

Figure 3.9. Plot of one-way ANOVA: number of unperformed activities, and staff ratio

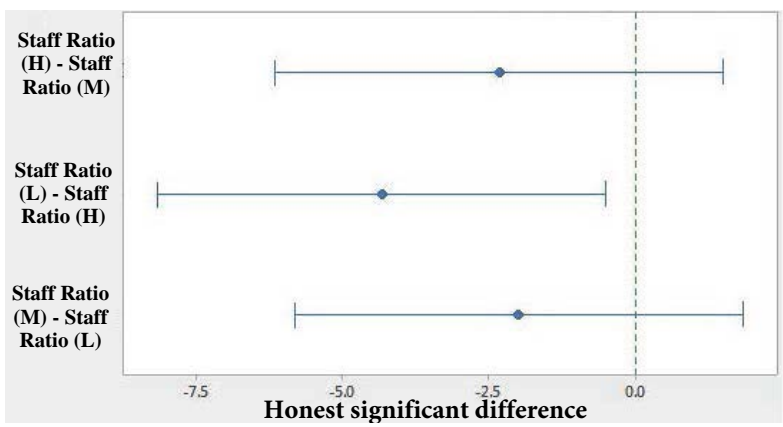

Figure 3.10. Plot of post-hoc analysis: effects of staff ratio on number of unperformed activities

Performing the experiment with regard to RQ2 confirms that staff ratio has significant effects on both available recovery time (\%) and the number of unperformed activities based on the results of ANOVA and the subsequent Tukey analysis. 


\subsection{RQ3: Effect of Acuity Indices}

\subsubsection{Effects on Available Recovery Time (\%)}

The results of ANOVA $(\mathrm{F}=1.42, \mathrm{p}=0.313)$ revealed a $\mathrm{p}$-value larger than 0.05 , which demonstrates an insignificant effect on recovery time due to varying acuity. Based on the result of the Tukey post-hoc analysis demonstrates that significant differences do not exist in available recovery time due to acuity indices (see Figure 3.11 and Figure 3.12).

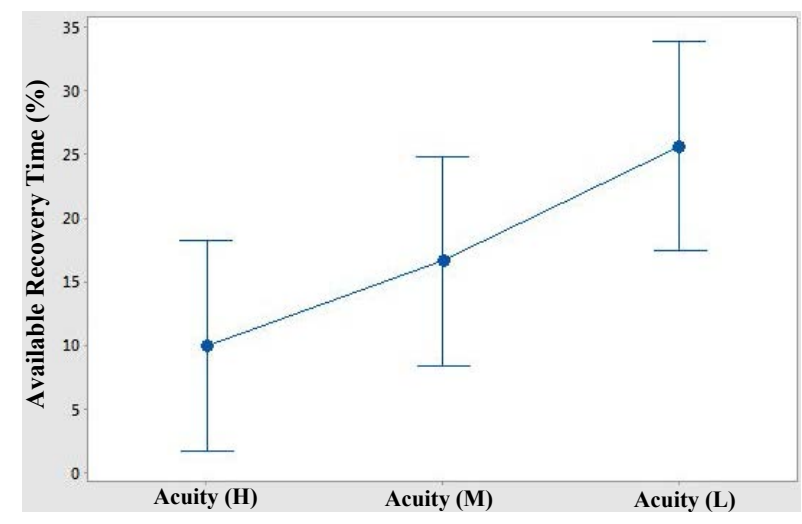

Figure 3.11. Plot of one-way ANOVA: available recovery time, and acuity

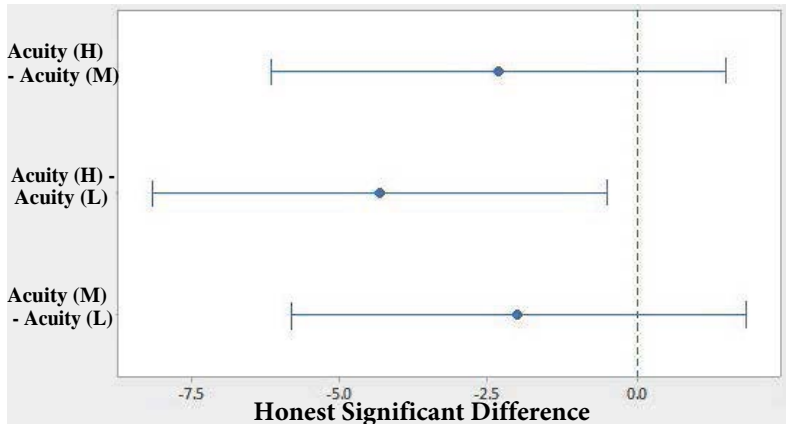

Figure 3.12. Plot of post-hoc analysis: effects of acuity indices on available recovery time 


\subsubsection{Effects on Number of Unperformed Activities}

The results of ANOVA $(\mathrm{F}=1.28, \mathrm{p}=0.344)$ revealed a $\mathrm{p}$-value that is larger than 0.05 , which demonstrates an insignificant effect due to varying acuity. The result of the Tukey posthoc analysis demonstrates that a significant difference does not exist in the number of unperformed activities due to acuity indices (see Figure 3.13 and Figure 3.14).

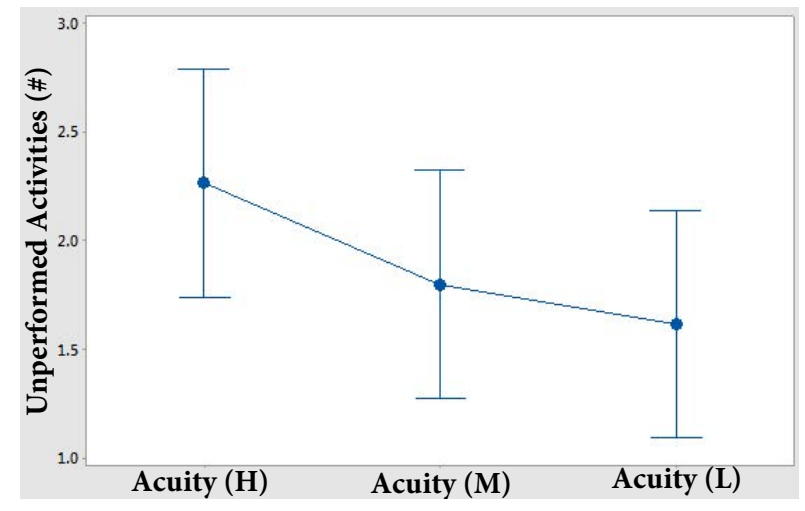

Figure 3.13. Plot of one-way ANOVA: number of unperformed activities, and acuity indices

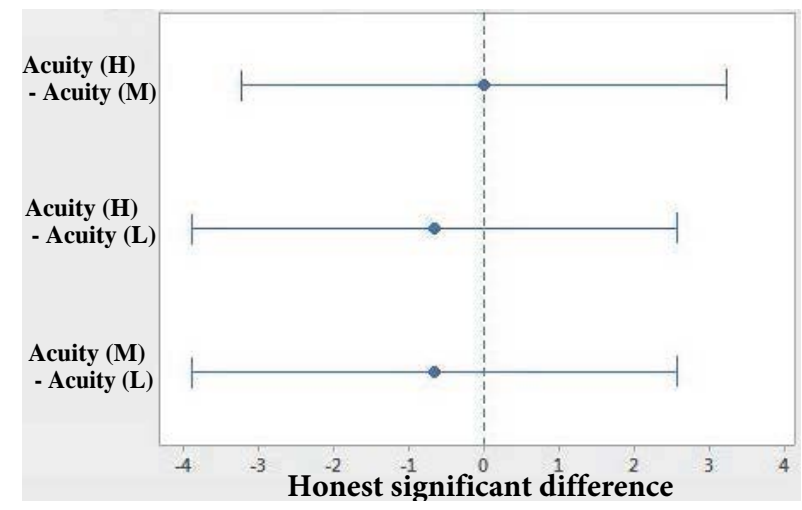

Figure 3.14. Plot of post-hoc analysis: effects of acuity indices on number of unperformed activities

By performing the experiment with regard to RQ3, it can be understood that the acuity indices have insignificant effects on both available recovery time (\%) and number of unperformed activities based on the results of ANOVA and subsequent Tukey analysis. However, it should be noted that acuity indices still exert influence, though insignificant in comparison to staff ratio, on both available recovery time and number of unperformed activities. 


\section{Chapter 4}

\section{Discussion}

The methods and results for each research question are discussed in this chapter. Moreover, time and effort related issues and obstacles are detailed such as lack of familiarity with the ABM premise, scarcity of similar studies in healthcare systems, and finally, the general lack of sufficient documentation materials for ABM. The chapter ends with the suggestions for future research.

\subsection{RQ1: ABM and the Virtual Unit}

Substantial work intensification has been observed in nearly all fields of industry and service. By combining engineering and human factor approaches, it is possible to control and mitigate the side effects of boundless work intensification through analysis of the routines and recovery times of personnel. This research was successful in achieving its aims regarding testing the feasibility of using ABM to analyze the workload of nurses in inpatient units. Not only was the model created based on empirical data, the virtual unit can be altered and combined with other virtual departments or clinics with the possibility of being used with other simulation approaches such as DES. 
Computer science and related simulation approaches are relatively new compared to their mathematical predecessors, and wider perspectives and opportunities exist for human factor research via computerized simulations. ABM was an appropriate approach to study inpatient healthcare units but drawbacks were apparent. Studying healthcare systems with many details and strict routines has revealed that $\mathrm{ABM}$ has obstacles compared with other approaches such as DES, which are typically used for looking for a best sequence or schedule of routines to decrease delays while downsizing staff levels. ABM has not yet achieved an equivalent capability due to its underlying premise of high abstraction and individualization. In addition, the focus of similar research has been on outpatient units such as EDs or ERs (Bonabeau 2002). It should be noted for inpatient units such as the selected unit in this investigation, the level of heterogeneity of agents is lower than it is with outpatient units (Günal \& Pidd 2007). This issue did not reveal itself in the model initiation stage but became evident during the model translation, which prevented the use of statistical distributions for activity durations. This was due to the nature of the acquired GRASP metrics, which were strictly defined; the noises and disruptions were already eliminated from the data. This research tried to address this drawback by having acuity indices and by adding tools that enabled the researcher to manually create disruptions in the system, e.g. adding/removing patients while running the simulation.

AnyLogic was selected as the ABM software to create the virtual unit due to the benefits described in Section 2.2.1. By using commercialized software, the researcher could focus on investigation with regard to research questions and avoid time and effort obstacles. However, as the investigation progressed, several disadvantages of relying on developing software were found. The lack of sufficient documentation (hard copy or online) for ABM and AnyLogic software, in comparison with DES and Arena software, made the investigation much more challenging and shifted the focus to JAVA programming as the substitute. Moreover, run-time of ABM model created in this investigation was slow (approximately eight hours for each 
replication for six months period of time for the virtual unit) due to excessive usage and strong dependency to the researcher's computer capacity i.e., random access memory (RAM), and this problem was aggravated by the fact that several iterations were performed. However, it should be noted that these issues are commonplace in nearly all ABM software. Other academic researchers have addressed these disadvantages by switching to object-oriented programming languages such as $\mathrm{C}++$, JAVA, or Python, which create the underlying platforms behind many ABM software products (Barnes et al. 2013).

\subsection{RQ2: Effects of Staff Ratio}

There is a paucity of ABM research addressing inpatient units, nurse/patient ratio, or the issue of acuity indices of patients to use for comparing the results of this investigation. The issue of staff ratios has been investigated and studied in several ABM studies for emergency departments (Bhandari et al. 2011, Laskowski \& Mukhi 2009). The focus in these studies was on cost efficiency or the number of waiting patients due to staff ratio alterations, and the agents were doctors and patients. Nurses, as an essential element in healthcare systems, were omitted. This research addressed this shortcoming by centring on the nurse as primary agent of this investigation to handle the issues of nurses' well-being (recovery time) and quality of care to patients simultaneously. This investigation found staff ratio has significant effects on available recovery time and the number of unperformed activities. This is consistent with other relevant ABM studies that have already pointed out the noteworthy staff ratio effects on quality of care; for instance, Laskowski \& Mukhi (2009) used ABM to study the effects of doctor/patient ratios and average number of unperformed activities in emergency departments. However, this investigation specifically demonstrated the effects of nurse/patient ratios on unperformed care activities as a measure of the quality of care, which is reconcilable with the findings of Aiken et al. (2002): the significant effects of staff ratio on 
on aspects of quality of care such as on-time delivery and also a higher level of well-being for nurses.

\subsection{RQ3: Effects of Acuity Indices}

The acuity indices of patients have been taken into consideration in some previous ABM studies for emergency departments (see Laskowski \& Mukhi (2009), Stainsby et al. (2009), and Taboada et al. (2011)). However, these applications dealt only with triage in emergency departments and were therefore related to dismissal rate. In this current study, the research shifted the focus onto the effects of acuity on human factors (such as recovery time) and quality of care to patients simultaneously by analyzing the number of unperformed activities and available recovery times for nurses in the virtual unit. However, the lack of significant effect by acuity indices could be due to the method used to assess the acuity indices in this investigation (HPPD) or the proportions used for each acuity level.

\subsection{Methodological Issues and Limitations}

The scarcity of relevant published work about how ABM and human factors function together was a limitation in the literature review stage of this investigation. To be more specific, as Neumann \& Medbo (2008) stated, using human factors principles even in DES is a new and growing approach, and this issue was also noticed in this investigation. Keywords such as "human factors" and "ABM" can be hard to find together in academic literature databases. As the rare examples of usage of ABM in human factors, see Sammarco et al.'s (2014) research on assembly flow shop, and Jiang \& Hu's (2010) study with regard to employee interaction with dynamic tasks.

With regard to in-data, as mentioned in Chapter 2, this research acquired and studied 
GRASP metrics as quantitative data from the selected unit. GRASP data consists of established, well-organized, validated metrics for the workload of nurses, recorded and analyzed by the quality assurance department of the designated hospital. However, the GRASP data from the selected unit had its own drawbacks: the names or frequencies of several activities were missing, and the data was acquired over a period of only one month. The limited completeness of the data as recorded by the unit of GRASP data, due to low-level participation of employees, decreased the quality of the data that was received. Moreover, with regard to acuity levels of patients, as one of the independent variables, other types of inpatient units such as surgical could demonstrate more impact on patient care and workload of nurses (Blegen \& Vaughn 1998). Furthermore, GRASP data from only one nurse was acquired, which may have over-interpretation when the results were compared with similar studies in order to comprehend the nature of the data and verify the results of this investigation. On the other hand, by using validated metrics, this study was able to keep the focus on its core objective and activity and avoid distraction: investigating the use of $\mathrm{ABM}$ in the inpatient healthcare system. 


\section{Chapter 5}

\section{Conclusion}

With regard to RQ1, this study has successfully created and adapted a virtual clinical unit, and the analysis of the results in the preceding chapters have shown the capacity and power of ABM for use as a method in studies of inpatient units. This investigation created a customizable virtual unit that can be adapted to different clinical units or even expanded to cover a full hospital to study the relationship between human factors and quality of service. With regard to RQ2, this investigation showed that staff ratio has substantial effects on both available recovery time and the number of unperformed activities in the simulation modeling. With regard to RQ3, acuity indices showed no significant effect on available recovery time or the number of unperformed activities in comparison to staff ratio in the simulation modeling. By adding the human factors related-metrics to the ABM simulation, the virtual unit has the capacity to be used as a tool to enable managers and engineers to better comprehend the effects of proposed system changes on the health of nurses and patient care quality and to avoid basing their decision only on the engineering aspects of design. 


\subsection{Contributions}

The novel contribution of this investigation is fourfold. The investigation demonstrated that $\mathrm{ABM}$ can be utilized to create a virtual unit to comprehend and analyze: $(a)$ the level of available recovery time for nurses, (b) the number of unperformed nursing care activities,

(c) the suitability of its use with an inpatient unit, and (d) the role of the nurses as primary caregivers to patients. This research demonstrated the power and research opportunities of ABM in nurses' well-being studies and enhancement of quality of care to patients. In a broader outlook, the virtual unit, as a customizable model, can be used by managers and engineers to predict results of their decisions from two perspectives simultaneously: human factors and quality of service.

\subsection{Future Studies}

This investigation did not consider the possibility of interaction between patients and other types of agents besides nurses. By including the results of interactions with other staff, the model could produce more meaningful results by examining the impact of all healthcare providers and on other indices of quality of care. Moreover, the model did not consider the length of stay of patients since this is more fitting to a continuous feedback loop modelling approaches such as system dynamics (Rohleder et al. 2007). Utilizing RFID tags is a well-established technique in ABM studies and can facilitate a better data collection and validation processes (Laskowski \& Mukhi 2009, Zhang et al. 2010). Many ABM studies of emergency departments have already used RFID tags on patients to observe and analyze their behaviours such as walking patterns; this approach may be adapted to movements of nurses in inpatient units.

To compensate for its slow run time, ABM should be run on new generations of computer 
processing units such as a Graphics Processing Unit (GPU). AnyLogic software has the power to be connected to databases such as Oracle and Microsoft MySQL which can help the data collection of nursing metrics. Some ABM studies, such as Bhandari et al. (2011) and Laskowski \& Mukhi (2009), addressed the number of doctors on call in an emergency room from a cost efficiency perspective; this approach can also be adapted to the created virtual unit but this time based on nurse/patient ratio in an inpatient unit.

As it has been stated in Chapter 3, the proposed virtual unit is capable to estimate available recovery time. Thus, it has the potential to reckon the threshold values for Maximum Voluntary Contraction (MVC) or Maximum Acceptable Effort (MAE) for caregivers and be used in accumulated muscular fatigue studies, Rose et al. (2011) or Potvin (2011), or to enhance the muscular fatigue recovery models, Rohmert (1973) or Rose et al. (1992). It can lead to a novel approach in occupational health studies and musculoskeletal disorder researches. 
Appendices 


\section{Appendix A}

\section{Pseudocodes}

The object oriented codes were done in JAVA language within AnyLogic Software environment.

\section{A.1 Code 01}

Function: The code 01 checks if the beds in the north wing of the clinic are vacant or not.

Output: The patient will be directed to a random vacant bed. The record or this inspection and movement is recorded.

int $\mathrm{j}=0$;

int $\mathrm{d}=0$;

Random random = new Random();

outerloop:

while $(\mathrm{d}==0)$

$\mathrm{j}=$ random.nextInt(collectBedsThree.length-1);

if (bedsThree[j] == false)

bedsThree[j]= true; 
break outerloop;

setXY(collectBedsThree[j],120);

traceln ("Index Three"+j);

\section{A.2 Code 02}

Function: The code 02 checks if the beds in the east wing of the clinic are vacant or not.

Output: The patient will be directed to a random vacant bed. The record or this inspection and movement is recorded.

int $\mathrm{d}=0$;

int $\mathrm{j}=0$;

Random random = new Random();

outerloop:

while $(\mathrm{d}==0)$

$\mathrm{j}=$ random.nextInt(collectBedsTwo.length-1);

if (bedsTwo[j]==false)

break outerloop;

bedsTwo $[\mathrm{j}]=$ false;

setXY(575,collectBedsTwo[j]);

setRotation(119.5);

traceln("Index Two"+j); 


\section{A.3 Code 03}

Function: The code 03 checks if the beds in the east south of the clinic are vacant or not.

Output: The patient will be directed to a random vacant bed. The record or this inspection and movement is recorded.

int $\mathrm{j}=0$;

int $\mathrm{d}=0$;

Random random = new Random();

outerloop:

while $(\mathrm{d}==0)$

$\mathrm{j}=$ random.nextInt(collectBedsOne.length-1);

if (bedsOne $[\mathrm{j}]==$ false)

break outerloop;

bedsOne $[\mathrm{j}]$ = true;

setXY(collectBedsOne[j],592);

setRotation(169.5);

traceln("Index One"+j);

\section{A.4 Code 04}

Function: The code 04 determines the number of patients in the clinic.

Output: It sets a lower bound for the number of patients.

int jk = get-Main().patients.size();

if $(\mathrm{jk}<12)$

get-Main().add-patients(); 


\section{A.5 Code 05}

Function: The code 05 assess the maximum capacity of clinic, i.e. 30 beds.

Output: It sets the upper bound for the number of admitted patients.

int $\mathrm{kj}$ = patients.size ();

if $(\mathrm{kj}<30)$

add-patients();

else

patientMax = true;

if (patients.size ()$<30$ )

patientMax = false;

\section{A.6 Code 06}

Function: The code 06 generates the intervention between the agents: nurse and patient.

Output: The request for nursing care activity is created and sent to the queue.

public class NurseRequest extends Entity implements java.io.Serializable

Patient patient;

public NurseRequest()

public NurseRequest(Patient patient)

this.patient $=$ patient;

@Override

public String toString() 
return

"patient = " + patient +" ";

Based on Borshchev (2013) and AnyLogic Software JAVA libraries.

\section{A.7 Code 07}

Input: The requested nursing care activity.

Output: The nursing care activity is assigned to a specific available nurse.

public class TreatmentRequest extends Entity implements java.io.Serializable

Patient patient;

public TreatmentRequest()

public TreatmentRequest(Patient patient)

this.patient $=$ patient;

@Override

public String toString()

return

"patient = " + patient +" ";

Based on Borshchev (2013) and AnyLogic Software JAVA libraries. 


\section{A.8 Code 08}

Input: The nursing care activity requested by an agent, i.e. patient. Output: The assigned nurse delivers the requested care: nutrition. public class Nutrition extends Entity implements java.io.Serializable Patient patient; public Nutrition()

public Nutrition(Patient patient) this.patient $=$ patient;

@Override

public String toString()

return

"patient = " + patient +" ";

Based on Borshchev (2013) and AnyLogic Software JAVA libraries.

\section{A.9 Code 09}

Input: The nursing care activity requested by an agent, i.e. patient. Output: The assigned nurse delivers the requested care: hygiene. public class Hygiene extends Entity implements java.io.Serializable Patient patient;

public Hygiene() 
public Hygiene(Patient patient)

this.patient $=$ patient;

@Override

public String toString()

return

"patient $=$ " + patient $+"$;

Based on Borshchev (2013) and AnyLogic Software JAVA libraries.

\section{A.10 Code 10}

Input: The nursing care activity requested by an agent, i.e. patient.

Output: The assigned nurse delivers the requested care: elimination.

public class Elimination extends Entity implements java.io.Serializable

Patient patient;

public Elimination()

public Elimination(Patient patient)

this.patient = patient;

@Override

public String toString()

return 
"patient = " + patient +" ";

Based on Borshchev (2013) and AnyLogic Software JAVA libraries.

\section{A.11 Code 11}

Input: The nursing care activity requested by an agent, i.e. patient.

Output: The assigned nurse delivers the requested care: treatments.

public class Treatments extends Entity implements java.io.Serializable

Patient patient;

public Treatments()

public Treatments(Patient patient)

this.patient $=$ patient;

@Override

public String toString()

return

"patient = " + patient +" ";

Based on Borshchev (2013) and AnyLogic Software JAVA libraries. 


\section{A.12 Code 12}

Input: The nursing care activity requested by an agent, i.e. patient.

Output: The assigned nurse delivers the requested care: other direct activities.

public class Others extends Entity implements java.io.Serializable

Patient patient;

public Others ()

public Others(Patient patient)

this.patient $=$ patient;

@Override

public String toString()

return

"patient = " + patient +" ";

Based on Borshchev (2013) and AnyLogic Software JAVA libraries.

\section{A.13 Code 13}

Input: The nursing care activity requested by an agent, i.e. patient.

Output: The assigned nurse delivers the requested care: non-direct activities.

public class NonDir extends Entity implements java.io.Serializable

Patient patient;

public NonDir() 
public NonDir(Patient patient)

this.patient $=$ patient;

@Override

public String toString()

return

"patient = " + patient +" ";

Based on Borshchev (2013) and AnyLogic Software JAVA libraries.

\section{A.14 Code 14}

Input: The nursing care activity requested by an agent, i.e. patient.

Output: The assigned nurse delivers the requested care: medication.

public class Medication extends Entity implements java.io.Serializable

Patient patient;

public Medication()

public Medication(Patient patient)

this.patient $=$ patient;

@Override

public String toString()

return 
"patient = " + patient +" ";

Based on Borshchev (2013) and AnyLogic Software JAVA libraries.

\section{A.15 Code 15}

Input: The nursing care activity requested by an agent, i.e. patient.

Output: The assigned nurse delivers the requested care: vascular access.

public class Vascular extends Entity implements java.io.Serializable

Patient patient;

public Vascular()

public Vascular(Patient patient)

this.patient $=$ patient;

@Override

public String toString()

return

"patient = " + patient +" ";

Based on Borshchev (2013) and AnyLogic Software JAVA libraries. 


\section{A.16 Code 16}

Input: The nursing care activity requested by an agent, i.e. patient.

Output: The assigned nurse delivers the requested care: vital signs.

public class Vital extends Entity implements java.io.Serializable

Patient patient;

public Vital()

public Vital(Patient patient)

this.patient $=$ patient;

@Override

public String toString()

return

"patient = " + patient +" ";

Based on Borshchev (2013) and AnyLogic Software JAVA libraries. 


\section{Appendix B}

\section{Output Analysis}

\begin{tabular}{ccccc}
\hline Mean & Standard Deviation & Confidence Interval & Accuracy & Initial number of replications \\
\hline 16 & 2.1 & $95 \%$ & 1.6 & 3 \\
\hline
\end{tabular}

\begin{tabular}{ccc}
\hline Replication & ${\text { T } \text { value }_{(\alpha / 2, R-1)}}$ & $\left(T * S D /\right.$ accuracy $^{2}$ \\
\hline 4 & 3.18 & 6.29 \\
5 & 2.78 & 4.69 \\
6 & 2.57 & 3.96 \\
7 & 2.45 & 3.55 \\
8 & 2.36 & 3.29 \\
9 & 2.31 & 3.11 \\
\hline $\mathbf{1 0}$ & $\mathbf{2 . 2 6}$ & $\mathbf{2 . 9 8}$ \\
\hline 11 & 2.23 & 2.80 \\
\hline
\end{tabular}

Table B.1. The minimum number of replications

\begin{tabular}{|c|c|c|c|c|}
\hline Run \#1 & Run \#2 & Run \#3 & Run \#4 & Run \#5 \\
\hline 16 & 7 & 15 & 15 & 17 \\
\hline Run \#6 & Run \#7 & Run \#8 & Run \#9 & Run \#10 \\
\hline \multirow[t]{3}{*}{16} & 17 & 17 & 15 & 16 \\
\hline & & Mean | & & \\
\hline & & \begin{tabular}{l|}
$16 \quad \mid$ \\
\end{tabular} & & \\
\hline
\end{tabular}

Table B.2. High \& High - Available Recovery Time (\%) 


\begin{tabular}{ccccc}
\hline Run \#1 & Run \#2 & Run \#3 & Run \#4 & Run \#5 \\
\hline 21 & 22 & 18 & 26 & 24 \\
\hline \hline Run \#6 & Run \#7 & Run \#8 & Run \#9 & Run \#10 \\
\hline 24 & 19 & 26 & 28 & 22 \\
\hline \multirow{5}{*}{} & \multicolumn{2}{c}{ Mean } & SD \\
\cline { 2 - 3 } & \multicolumn{2}{c|}{23} & 2.29 \\
\hline
\end{tabular}

Table B.3. High \& Medium - Available Recovery Time (\%)

\begin{tabular}{|c|c|c|c|c|}
\hline Run \#1 & Run \#2 & Run \#3 & Run \#4 & Run \#5 \\
\hline 36 & 31 & 38 & 37 & 36 \\
\hline Run \#6 & Run \#7 & Run \#8 & Run \#9 & Run \#10 \\
\hline \multirow[t]{3}{*}{36} & 32 & 27 & 28 & 29 \\
\hline & & Mean | & SD & \\
\hline & & 33 & 1.7 & \\
\hline
\end{tabular}

Table B.4. High \& Low - Available Recovery Time (\%)

\begin{tabular}{|c|c|c|c|c|}
\hline Run \#1 & Run \#2 & Run \#3 & Run \#4 & Run \#5 \\
\hline 36 & 41 & 37 & 40 & 38 \\
\hline Run \#6 & Run \#7 & Run \#8 & Run \#9 & Run \#10 \\
\hline \multirow[t]{3}{*}{41} & 39 & 38 & 30 & 40 \\
\hline & & Mean | & & \\
\hline & & 38 & & \\
\hline
\end{tabular}

Table B.5. Medium \& High - Available Recovery Time (\%)

\begin{tabular}{|c|c|c|c|c|}
\hline Run \#1 & Run \#2 & Run \#3 & Run \#4 & Run \#5 \\
\hline 40 & 39 & 44 & 46 & 49 \\
\hline Run \#6 & Run \#7 & Run \#8 & Run \#9 & Run \#10 \\
\hline \multirow[t]{3}{*}{43} & 41 & 48 & 40 & 40 \\
\hline & & Mean I & & \\
\hline & & $43 \quad$ & & \\
\hline
\end{tabular}

Table B.6. Medium \& Medium - Available Recovery Time (\%)

\begin{tabular}{|c|c|c|c|c|}
\hline Run \#1 & Run \#2 & Run \#3 & Run \#4 & Run \#5 \\
\hline 48 & 49 & 42 & 51 & 47 \\
\hline Run \#6 & Run \#7 & Run \#8 & Run \#9 & Run \#10 \\
\hline \multirow[t]{3}{*}{50} & 46 & 47 & 44 & 44 \\
\hline & & Mean I & & \\
\hline & & $47 \quad$ & & \\
\hline
\end{tabular}

Table B.7. Medium \& Low - Available Recovery Time (\%) 


\begin{tabular}{|c|c|c|c|c|}
\hline Run \#1 & Run \#2 & Run \#3 & Run \#4 & Run \#5 \\
\hline 44 & 43 & 42 & 43 & 47 \\
\hline Run \#6 & Run \#7 & Run \#8 & Run \#9 & Run \#10 \\
\hline \multirow[t]{3}{*}{40} & 48 & 47 & 44 & 44 \\
\hline & & Mean | & & \\
\hline & & 44 & & \\
\hline
\end{tabular}

Table B.8. Low \& High - Available Recovery Time (\%)

\begin{tabular}{|c|c|c|c|c|}
\hline Run \#1 & Run \#2 & Run \#3 & Run \#4 & Run \#5 \\
\hline 52 & 54 & 50 & 45 & 46 \\
\hline Run \#6 & Run \#7 & Run \#8 & Run \#9 & Run \#10 \\
\hline \multirow[t]{3}{*}{54} & 52 & 53 & 54 & 51 \\
\hline & & Mean | & & \\
\hline & & 51 & & \\
\hline
\end{tabular}

Table B.9. Low \& Medium - Available Recovery Time (\%)

\begin{tabular}{|c|c|c|c|c|}
\hline Run \#1 & Run \#2 & Run \#3 & Run \#4 & Run \#5 \\
\hline 50 & 58 & 61 & 58 & 59 \\
\hline Run \#6 & Run \#7 & Run \#8 & Run \#9 & Run \#10 \\
\hline \multirow[t]{3}{*}{58} & 59 & 62 & 62 & 61 \\
\hline & & Mean | & & \\
\hline & & 59 & & \\
\hline
\end{tabular}

Table B.10. Low \& Low - Available Recovery Time (\%) 


\section{Bibliography}

Aiken, L., Clarke, S., Sloane, D., Lake, E. \& Cheney, T. (2008), 'Effects of hospital care environment on patient mortality and nurse outcomes', Journal Nursing Administration 35(8), 223-229.

Aiken, L., Clarke, S., Sloane, D., Sochalski, J. \& Silber, J. (2002), 'Hospital nurse staffing and patient mortality, nurse burnout, and job dissatisfaction', Journal of the American Medical Association 288(16), 1987-1993.

Andersson, R. (2007), Quality-driven Logistics, PhD thesis, Division of Logistics and Transportation, Department of Technology Management and Economics, Charlmers University.

AnyLogic (2015), 'Healthcare and pharmaceuticals simulation'.

URL: http://www.anylogic.com/consulting/healthcare-and-pharmaceuticals

Banks, J. (2010), Discrete-event System Simulation, Prentice Hall. ISBN: 9780136062127.

Barnes, S., Golden, B. \& Price, S. (2013), 'Applications of agent-based modelling and simulation to healthcare operations management', International Series in Operations Research \& Management Science 184, 45-74.

Bergman, B. \& Klefsjö, B. (2009), Quality: From Customer Needs to Customer Satisfaction, Studentlitteratur AB. ISBN: 9144041667. 
Bhandari, G., Kobti, Z., Snowdon, A., Nakhwal, A., Rahaman, S. \& Kolga, C. (2011), Agentbased modelling and simulation as a tool for decision support for managing patient falls in a dynamic hospital setting, in D. Schuff, D. Paradice, F. Burstein, D. J. Power \& R. Sharda, eds, 'Decision Support', Vol. 14 of Annals of Information Systems, Springer New York, pp. 149-162. ISBN: 9781441961808.

Blegen, M. \& Vaughn, T. (1998), 'A multisite study of nurse staffing and patient occurrences', Nursing Economics 16(4), 196-203.

Bonabeau, E. (2002), 'Agent-based modelling: Methods and techniques for simulating human systems', Bonabeau 99(3), 7280-7287.

Borshchev, A. (2013), The Big Book of Simulation Modelling: Multimethod Modelling with AnyLogic 6, AnyLogic North America. ISBN: 0989573176.

Cassandras, C. \& Lafortune, S. (2009), Introduction to Discrete Event Systems, $2^{\text {nd }}$ edn, Springer. ISBN: 9780387686127.

Charfeddine, M. \& Montreuil, B. (2008), Toward a conceptual agent-based framework for modelling and simulation of distributed healthcare delivery systems, Technical report, Interuniversity Research Centre on Enterprise Network, Logistics and Transportation, Canada.

Chaudhry, B., Wang, J., Wu, S., Maglione, M., Mojica, W., Roth, E., Morton, S. \& Shekelle, P. (2006), 'Systematic review: Impact of health information technology on quality, efficiency, and costs of medical care', Annals of Internal Medicine 144(10), 742-752.

Cimete, G., Gencalp, N. \& Keskin, G. (2003), 'Quality of life and job satisfaction of nurses', Nursing Care Quality 18(2), 151-158. 
Cimiotti, J. P., Aiken, L. H., Sloane, D. M. \& Wu, E. S. (2012), 'Nurse staffing, burnout, and healthcare-associated infection', American Journal of Infection Control 40(6), 486-490.

Collins, A. \& Noble, R. (1992), 'Hospital rightsizing: In line with long-term strategies and economic realities', Healthcare Management Forum 5(1), 4-10.

Dahlgaard, J. J., Pettersen, J. \& Dahlgaard-Park, S. M. (2011), 'Quality and Lean Healthcare: A system for assessing and improving the health of healthcare organizations', Total Quality Management and Business Excellence 22(6), 673-689.

Duguay, C. \& Chetouane, F. (2007), 'Modelling and improving emergency department systems using discrete event simulation', Computer Science and Software Engineering 1(63), 311320.

Dul, J. \& Neumann, W. P. (2006), The Strategic Business Value of Ergonomics, Vol. The $16^{\text {th }}$ World Congress on Ergonomics, Meeting Diversity in Ergonomics, Maastricht, Netherlands.

Dul, J. \& Neumann, W. P. (2009), 'Ergonomics Contributions to Company Strategies', Applied Ergonomics 40(4), 745-752.

Enthoven, A. \& Tollen, L. (2005), 'Competition in healthcare: It takes systems to pursue quality and efficiency', Health Affairs 24(1), 420-433.

Farrington, M., .Trundle, C., Redpath, C. \& Anderson, L. (2000), 'Effects on nursing workload of different methicillin-resistant staphylococcus aureus (MRSA) control strategies', Journal of Hospital Infection 46(2), 118-122.

Gaba, D. (2004), 'The future vision of simulation in healthcare', The international journal of healthcare improvement 13(1), 12-22. 
Gingras, J., De Jonge, L. \& Purdy, N. (2010), 'Prevalence of dietitian burnout', Journal of Human Nutrition and Dietetics 23(3), 238-243.

Grandjean, E. \& Kroemer, K. (1997), Fitting The Task To The Human, $5^{\text {th }}$ edn, CRC Press. ISBN: 9780748406654.

GRASP Systems International (2012), Unit reference manual, Technical report, University Health Network.

Günal, M. \& Pidd, M. (2007), 'Interconnected des models of emergency, outpatient, and inpatient departments of a hospital', Simulation Conference 1(2), 1461-1466.

Günal, M. \& Pidd, M. (2010), 'Discrete event simulation for performance modelling in healthcare: a review of the literature', Journal of Simulation 4(1), 42-51.

Hall, D. (2004), 'Work-related stress of registered nurses in a hospital setting', Journal for Nurses in Staff Development 20(1), 6-14.

Harvey, C., Buckley, C., Forrest, R., Roberts, J., Searle, J., Meyer, A. \& Thompson, S. (2015), 'Aberrant work environments, rationed care as system failure or missed care as skills failure?', GSTF Journal of Nursing and Healthcare 2(1), 72-80.

Harvey, T., Pati, D., Evans, J. \& Waggener, L. (2006), Inpatient unit design: defining the design characteristics of a successful adaptable unit, AIA Report on University Research.

Hongqiao, Y., Xihua, L., Fei, W. \& Weizi, L. (2009), 'Multi-agent based modelling and simulation of complex system in hospital', $16^{\text {th }}$ International Conference of Industrial Engineering and Engineering Management IE EM(09), 1759-1763.

IBRD (2015), 'Health expenditure, total (\% of gdp)'. 
International Ergonomics Association (2015), What is Ergonomics, IEA.

URL: $h t t p: / / w w w . i e a . c c / w h a t s /$

James, F. (2006), Statistical Methods in Experimental Physics, World Scientific Publishing.

Jiang, G. \& Hu, B. (2010), 'Agent-based simulation approach to understanding the interaction between employee behaviour and dynamic tasks', SIMULATION 87(5), 407-422.

Kalisch, B. J., Landstrom, G. \& Williams, R. A. (2009), 'Missed nursing care: errors of omission', Nursing Outlook 57(1), 3-9.

Karnon, J. (2003), 'Alternative decision modelling techniques for the evaluation of healthcare technologies: Markov processes versus discrete event simulation', Health economics 12(10), 837-848.

Karsh, B., Holden, R., Alper, S. \& Or, C. (2006), 'A human factors engineering paradigm for patient safety: designing to support the performance of the healthcare professional', Quality Safety Health Care 1(1), 59-65.

Kudyba, S. (2001), Data Mining and Business Intelligence: A Guide to Productivity, Igi Global. ISBN: 1930708033.

Lang, T., Hodge, M. \& Olson, V. (2004), 'Nurse-patient ratios: A systematic review on the effects of nurse staffing on patient, nurse employee, and hospital outcomes', The Journal of Nursing Administration 34(8), 326-337.

Laschinger, H., Finegan, J. \& Shamian, J. (2001), 'The impact of workplace empowerment, organizational trust on staff nurses' work satisfaction and organizational commitment', Healthcare Management Review 26(3), 7-23.

Laskowski, M. \& Mukhi, S. (2009), 'Agent-based simulation of emergency departments with patient diversion', Social Informatics and Telecommunications Engineering 1, 25-37. 
Lee, M. (2008), Identification of Clinical and Economic Effectiveness of Nursing Care for the Hospitalized Clients with Total Hip Replacement, University of Iowa. ISBN: 9780549950646.

Macy, M. \& Willer, R. (2002), 'From factors to actors: Computational sociology and agentbased modelling', Annual Review of Sociology 22(1), 143-166.

Montgomery, D. (2012), Introduction to Statistical Quality Control, $7^{\text {th }}$ edn, Wiley. ISBN: 0470169923.

Needleman, J., Buerhaus, P., Mattke, S., Stewart, M. \& Zelevinsky, K. (2002), 'Nurse-staffing levels and the quality of care in hospitals', New England Journal of Medicine 346(22), 17151722.

Neumann, W. P. \& Dul, J. (2010), 'Human Factors: spanning the gap between OM and HRM', International Journal of Operations \& Production Management 30(9), 923-950.

Neumann, W. P., Ekman, M. \& Winkel, J. (2009), 'Integrating ergonomics into production system development - the Volvo powertrain case', Applied Ergonomics 40(3), 527-537.

Neumann, W. P. \& Medbo, P. (2008), 'Integrating Human Factors into Discrete Event Simulations of Parallel Flow Strategies', Production Planning \& Control: The Management of Operations 2009(1), 3-16.

Nursing Task Force (1999), Good Nursing, Good Health : An Investment for the $21^{\text {st }}$ Century, Ministry of Health and Long-Term Care, Ontario, Canada.

Pan, X., Han, C., Dauber, K. \& Law, K. (2007), 'A multi-agent based framework for the simulation of human and social behaviours during emergency evacuations', AI and SOCIETY 22(2), 113-132. 
Potvin, J. R. (2011), 'Predicting Maximum Acceptable Efforts for Repetitive Tasks: An Equation Based on Duty Cycle', Human Factors: The Journal of the Human Factors and Ergonomics Society 54(2), 175-188.

Purdy, N. (2011), Effects of work environments on nursing and patient outcomes, University of Western Ontario, London, Ontario.

Purdy, N., Spence, L., Heather, K., Finegan, J., Kerr, M. \& Olivera, F. (2010), 'Effects of work environments on nurse and patient outcomes', Journal of Nursing Management 18(8), 901913.

Rafferty, A. M., Clarke, S. P., Coles, J., Ball, J., James, P., McKee, M. \& Aiken, L. H. (2007), 'Outcomes of variation in hospital nurse staffing in English hospitals: Cross-sectional analysis of survey data and discharge records', International Journal of Nursing Studies 44(2), 175-182.

Rohleder, T., Bischak, D. \& Baskin, L. (2007), 'Modelling patient service centres with simulation and system dynamics', Healthcare Management Science 10(1), 1-12.

Rohmert, W. (1973), 'Problems in determining rest allowances. part i: Use of modern methods to evaluate stress and strain in static muscular work', Applied Ergonomics 4, 91-95.

Rose, L., Ericsson, M., Glimskära, B., Nordgren, B. \& Örtengren, R. (1992), 'Ergo-index: Development of a model to determine pause needs after fatigue and pain reactions during work', Computer Applications pp. 461-468.

Rose, L., Neumann, W. P. \& Orrenius, U. (2011), 'Work environment and the bottom line: Survey of tools relating work environment to business results', Human Factors and Ergonomics in Manufacturing and Service Industries 23(5), 368-381.

Rossetti, M. (2010), Simulation Modelling and Arena, Wiley. ISBN: 0470097264. 
Sammarco, M., Fruggiero, F., Neumann, W. P. \& Lambiase, A. (2014), 'Agent-based modelling of movement rules in DRC systems for volume flexibility: human factors and technical performance', International Journal of Production Research 52(3), 633-650.

Schlesinger, S. (1989), 'Terminology model for credibility', Simulation 32(3), 103-104.

Shah, C. (2003), Public Health and Preventive Medicine in Canada, $5^{\text {th }}$ edn, Elsevier. ISBN: 9780920513934.

Sibbel, R. \& Urban, C. (2001), Agent-based modelling and simulation for hospital management, in N. Saam \& B. Schmidt, eds, ‘Cooperative Agents', Vol. 32 of Theory and Decision Library, Springer Netherlands, pp. 183-202.

Siebers, P., Macal, C., Garnett, J., Buxton, D. \& Pidd, M. (2010), 'Discrete-event simulation is dead, long live agent-based simulation!', Journal of Simulation 4, 204-210.

Slee, V., Slee, D. \& Schmidt, H. (2001), Slee's Healthare Terms, $4^{\text {th }}$ edn, Tringa Press. ISBN: 0763746150.

Stainsby, H., Taboada, M. \& Luque, E. (2009), 'Towards an agent-based simulation of hospital emergency departments', Services Computing, 2009. IEEE International Conference on SCC(09), 536-539.

Swisher, J. \& Jacobson, S. (2002), 'Evaluating the design of a family practice healthcare clinic using discrete-event simulation', Healthcare Management Science 5(2), 75-88.

Taboada, M., Cabrera, E., Iglesias, M. L., Epelde, F. \& Luque, E. (2011), 'An agent-based decision support system for hospitals emergency departments', Procedia Computer Science 4, 1870-1879.

Tesfatsion, L. (2003), 'Agent-based computational economics: modelling economies as complex adaptive systems', Information Sciences 149(4), 262-268. 
Tsuji, Y., Kawaguchi, T. \& Tanaka, T. (1993), 'Discrete particle simulation of two-dimensional fluidized bed', Powder Technology 77(1), 79-87.

van Vuuren, J., Burger, A. \& van der Merwe, M. (2012), 'An Asymptotic Analysis of the Evolutionary Spatial Prisoner's Dilemma on a Path', Discrete Applied Mathematics 160(15), 20752088.

Varoufakis, Y. \& Hargreaves-Heap, S. (2004), Game Theory: A Critical Introduction, $2^{\text {nd }}$ edn, Routledge.

Weiner, B. J., Alexander, J. A., Shortell, S. M., Baker, L. C., Becker, M. \& Geppert, J. J. (2006), 'Quality improvement implementation and hospital performance on quality indicators', Health Services Research 41(2), 307-334.

Wilde, B., Larsson, G., Larsson, M. \& Starrin, B. (1994), 'Quality of care', Scandinavian Journal of Caring Sciences 8(1), 39-48.

Yoder, E. (2010), 'Compassion fatigue in nurses', Applied Nursing Research 23(4), 191-197.

Yuan, S., Chou, M., Chen, C., Lin, Y., Chen, M., Liu, H. \& Kuo, H. (2011), 'Influences of shift work on fatigue among nurses', Journal of Nurse Management 19(3), 339-345.

Zhang, Y., Huang, G. Q., Qu, T. \& Ho, O. (2010), 'Agent-based workflow management for RFID-enabled real-time reconfigurable manufacturing', International Journal of Computer Integrated Manufacturing 23(2), 101-112. 